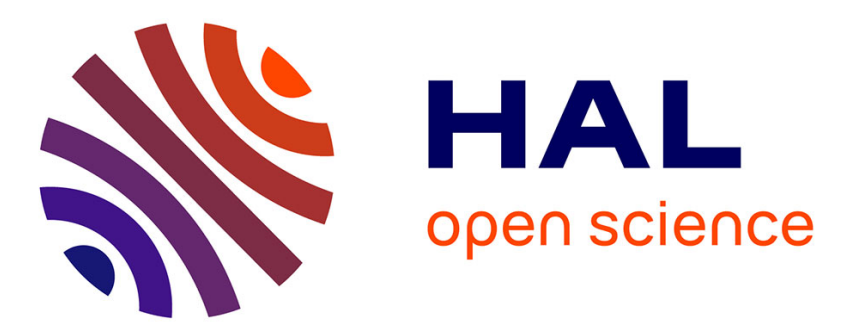

\title{
Combined voids size and shape effects on the macroscopic criterion of ductile nanoporous materials
}

\author{
Vincent Monchiet, Djimedo Kondo
}

\section{To cite this version:}

Vincent Monchiet, Djimedo Kondo. Combined voids size and shape effects on the macroscopic criterion of ductile nanoporous materials. International Journal of Plasticity, 2013, 43, pp.20-41. 10.1016/j.ijplas.2012.10.007 . hal-01165815

\section{HAL Id: hal-01165815 \\ https://hal.science/hal-01165815}

Submitted on 18 Mar 2021

HAL is a multi-disciplinary open access archive for the deposit and dissemination of scientific research documents, whether they are published or not. The documents may come from teaching and research institutions in France or abroad, or from public or private research centers.
L'archive ouverte pluridisciplinaire HAL, est destinée au dépôt et à la diffusion de documents scientifiques de niveau recherche, publiés ou non, émanant des établissements d'enseignement et de recherche français ou étrangers, des laboratoires publics ou privés. 


\title{
Combined voids size and shape effects on the macroscopic criterion of ductile nanoporous materials
}

\author{
Vincent MONCHIET ${ }^{\mathrm{a}}$, Djimedo KONDO ${ }^{\mathrm{b}}$ \\ ${ }^{a}$ Université Paris-Est, Modélisation et Simulation Multi Echelle, MSME UMR \\ 8208 CNRS, \\ 5 bd Descartes, 77454 Marne-la-Vallée, France \\ ${ }^{\mathrm{b}}$ Université Pierre et Marie Curie, Institut D'Alembert, UMR 7190 CNRS, \\ 4, Place Jussieu, 75252 Paris Cedex 05, France
}

\begin{abstract}
In this paper, we investigate the interfacial stress effects on the macroscopic yield function of ductile porous media containing nanosized spheroidal cavities. The solid matrix is assumed rigid-ideal plastic and of von Mises type with associated flow rule. We then perform limit analysis of a spheroidal unit cell containing a confocal spheroidal (prolate or oblate) cavity and subjected to arbitrary mechanical loadings. Voids size effects are captured by considering at the interface between the matrix and the cavity a surface stress model which relates the jump of the traction vector to the interfacial residual stress and interfacial plastic strain. This accounts for a thin shell of material in which occurs a strong plastic strain accumulation. We then provide a closed-form two-field based estimate of the overall dissipation which contains additional terms related to the interfacial plasticity. By taking advantage of this result, we derive parametric equations of the macroscopic yield surface of the nanoporous plastic material. The obtained estimates are assessed through comparisons with numerical data. Finally, it is shown that the resulting macroscopic criterion of the nanoporous material exhibits specific features such as (i) a dependence of the yield stress on the size of the spheroidal nanovoids, (ii) asymmetry between the yield stress in uniaxial tension and compression, (iii) more pronounced size effects for oblate voids than for prolate ones.
\end{abstract}

Key words: Ductile Materials, Yield Criterion, Voids Size Effects, Prolate or Oblate Cavities, Interfacial Stress.

Email addresses: vincent.monchiet@univ-mlv.fr (Vincent MONCHIET), djimedo.kondo@upmc.fr (Djimedo KONDO). 


\section{Introduction}

The elastic properties of solids are significantly affected by the presence of surfaces and interfaces (see $[68,13,10,41]$ for experimental evidence and $[48,14,11,59,66]$ for numerical results). Surface effects are attributed to the presence of few layers of atoms which experience a different local environment than atoms in the bulk and have a different equilibrium positions and energy. The classical threedimensional linear elasticity theory generally neglects these effects since the considered objects and structural elements are, at less, microsized but never of the size of one or few nanometers. For such nanosized objects, the interfacial effects become predominant since the area of surface per unit of volume is very high. Surface effects in standard elasticity theories are modeled by means of the Gurtin and Murdoch stress interface model $[36,37]$ which assumes a jump of the traction vector while the displacement field is considered continuous across the surface (see also Povstenko [58], Steigman and Ogden [62] for various extensions). The jump condition may comply with a generalized Young-Laplace equation which extends to solid-solid interface the well known equation that describes capillarity effects in fluid mechanics. Thus, the discontinuity of the traction vector consists of two parts, the first one is attributed to the presence of interfacial residual stresses, independent of the deformation, and the second one being related to elastic deformation of the interface. Note that various studies, based on ab initio calculations and molecular dynamics, have been recently proposed for the identification of interfacial elastic coefficients and the residual stress that occur at the free surface of a monocrystalline material $[48,61,38,71]$. Mention must be also done of studies evaluating the effects of surface free energy on the elastic behavior of nano-sized particles (see for instance [17]).

Gurtin type stress surface model has been used to investigate the inclusions size dependency of elastic properties of nanocomposites. For instance, [19,21,22] have proposed to generalize the fundamental framework of micromechanical procedure to take into account the surface/interface stress effect at the nanoscale. Sharma and Ganti [60] generalize the Eshelby inclusion problem by incorporating the interface stress. Other works have proposed to derive bounds for the size dependent effective properties of nanocomposites $[42,6,7]$. However, it can be noted that all these studies generally concern spherical or cylindrical nano-inhomogeneities since the solution of the corresponding Eshelby type problem is only known for this kind of inclusion shape. Mention has to be made of the study by $\mathrm{Ou}$ et al. $[54,55]$ who, by using displacement harmonic potential functions methods, attempted to derive the elastic fields around a nanosized spheroidal inclusion or cavity with surface effect. However, their studies do not deliver closed form or semi analytic expressions. In fact, a general micromechanics theory of elastic materials containing nanoinclusions, based on the solution of the Eshelby ellipsoidal inhomogeneity problem with 
interfacial stress, is still lacking and is not the subject of the present study.

Surface effects on the yield strength and plastic behavior of nano objects and nanostructured materials have also been observed (see for instance $[46,69]$ for experimental evidence and $[15,26,56,57,16,70,34]$ for numerical simulations). Few recent studies consider stress interfaces effects in the context of non-linear composites or nanoporous ductile materials [72-74,18,34,50]. In particular, by performing a limit analysis of a hollow sphere, Dormieux and Kondo [18] generalize the Gurson model [35] in order to predict void size effects. To this end, they make use of a plastic version of the Gurtin stress interface model (see for instance [50]) which relates the interfacial stress to the plastic deformation at the cavity surface. The resulting model shows a void size dependency of the macroscopic yield strength of nanoporous media: for nanosized spherical cavities, the strength domain appears to be significantly larger than that predicted by the Gurson model.

Alternatively, for porous media, non-local plasticity theories have been used in $[64,45,67,40,43,44]$ for the plastic behavior of the solid matrix instead of the von Mises model. In $[45,67]$, the authors extend the Rice-Tracey or the Gurson model by considering, for the solid matrix, the Taylor dislocation based theory of plasticity introduced by [28]. In [40,43,44], the size effect is captured by the Fleck \& Hutchinson phenomenological strain gradient plasticity theory [24] (see also [25] for a study of voids size effect on the plastic behavior of porous ductile media). It is interesting to note that the result obtained by Dormieux et al. [18] are qualitatively comparable to that earlier works mentioned above, which also predict an increase of the elastic domain by decreasing the size of the cavity. Still in the context of non-local plasticity theories, several studies are devoted to the study of combined voids shape and voids size effects (see for instance $[39,40,43,44])$. In all these studies, the void size effect is interpreted as the dependence of the solid matrix plasticity with an internal length scale which is physically attributed to the generation and the storage of geometrically necessary dislocations (Nye [53], Cottrell [12], Ashby [2], Fleck et al. [23], Gao et al. [27]). A priori, there is no direct link between the "interfacial stress" and the "non local approaches". However, and along the lines of Fleck and Hutchinson [24], microstructural effects of plastic media occur when the strain gradient are sufficiently large compared to dislocation spacing. Still, according to these authors, "the expanding void is surrounded by a shell of hardened material due to the presence of both strain and strain gradient". Note that, based on [50], the use of the plastic version of the stress interface model can be physically interpreted as the existence of a thin region surrounding the interface in which intense gradient of plastic deformation will occur. Interface stresses model can be seen as an alternative approach to account for plastic strain accumulation at the cavity surface.

Such approach has been developed by [18] who restrict their analysis to the 
case of spherical cavities. In the present study, we provide an extension of their approach to the case of spheroidal (prolate or oblate) cavities. By doing so, we also extend the classical work of Gologanu et al. [31,32] by simultaneously accounting for combined voids shape and void size effects. The paper is organized as follows. In section 2, we present the limit analysis of the hollow spheroid in the presence of stress interface. In section 3, we recall the trial velocity field already considered in $[31,32]$ from which is computed a compact expression of the interfacial plastic strain. A two-field estimate of the macroscopic dissipation, including new terms related to the stress interface, is derived in section 4 from which we determine the parametric equations of the macroscopic yield surface of the nanoporous medium (see section 5). Finally, in section 6 , we provide various illustrations of the combined voids shape and size dependency of the macroscopic criterion. In particular, the results of our two-field estimate are compared to data obtained by numerical computation of the macroscopic dissipation.

\section{The limit analysis accounting for interfacial stress}

\subsection{Description of the unit cell}

Let us consider a spheroidal cavity of semi-axes $a_{1}$ and $b_{1}$ embedded in a confocal spheroid of semi-axes $a_{2}$ and $b_{2}$ (see figure 1 ). The axis of the spheroids are aligned with $0 x_{3}$, where $\left(0, x_{1}, x_{2}, x_{3}\right)$ is a cartesian coordinate system of orthonormal basis $\left(\underline{e}_{1}, \underline{e}_{2}, \underline{e}_{3}\right)$.
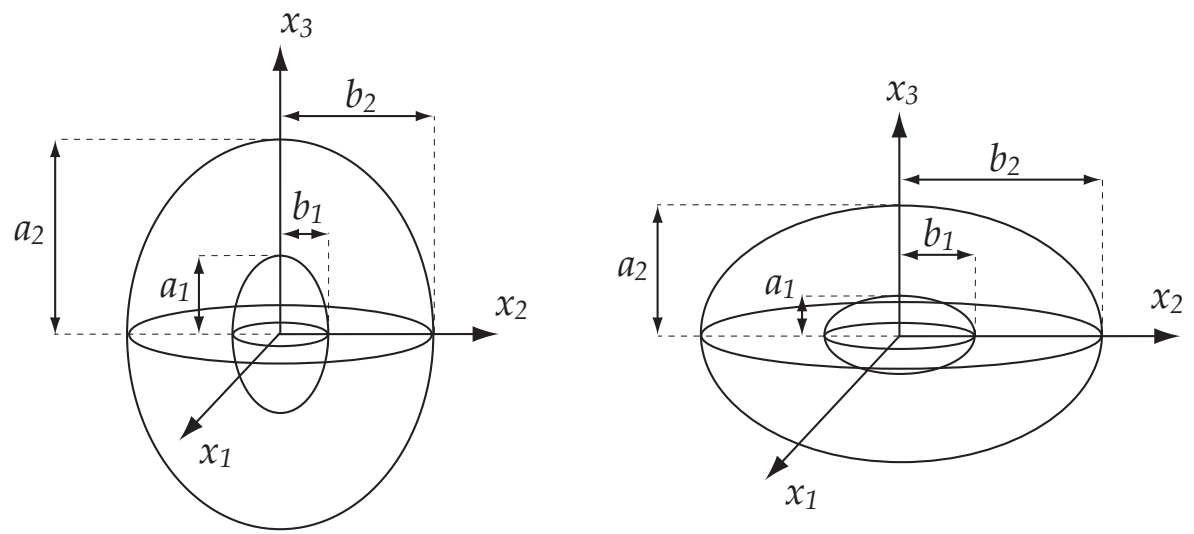

Fig. 1. The spheroidal unit cell with a confocal spheroidal void. Case of prolate cavity (at the left) and prolate cavity (at the right).

The assumption of confocality has been made in order to simplify analytical computation of the macroscopic criterion in the framework of limit analysis 
methods. Note that it is the unique one that has been considered in literature dealing with Gurson type approach. Despite its simplicity, the confocal cell allows to represent microstructures of the type "hollow spheroid assemblages" (see for instance Benveniste and Milton [3]).

The volume of the cavity is $V_{1}=4 \pi a_{1} b_{1}^{2} / 3$ while the total volume of the unit-cell is $V_{2}=4 \pi a_{2} b_{2}^{2} / 3$. The shape of the cavity is described by the aspect ratio $a_{1} / b_{1}$, with $a_{1}>b_{1}$ corresponding to a prolate cavity while $b_{1}>a_{1}$ to an oblate one. Let us denote by $c$ the focal distance and by $e_{1}$ the void eccentricity, defined by:

$$
\begin{array}{llll}
c=\sqrt{a_{1}^{2}-b_{1}^{2}}=\sqrt{a_{2}^{2}-b_{2}^{2}} & e_{1}=\frac{c}{a_{1}} & e_{2}=\frac{c}{a_{2}} & \text { (prolate) } \\
c=\sqrt{b_{1}^{2}-a_{1}^{2}}=\sqrt{b_{2}^{2}-a_{2}^{2}} & e_{1}=\frac{c}{b_{1}} & e_{2}=\frac{c}{b_{2}} & \text { (oblate })
\end{array}
$$

We will use both cylindrical coordinates $\rho, \theta, \mathrm{z}$ and $\left(\underline{e}_{\rho}, \underline{e}_{\theta}, \underline{e}_{z}\right)$ the associated orthonormal basis and the classical spheroidal coordinates $\lambda, \varphi, \theta$ (associated orthogonal basis $\left(\underline{e}_{\lambda}, \underline{e}_{\phi}, \underline{e}_{\theta}\right)$ defined by:

$$
\left\{\begin{array} { l } 
{ x _ { 1 } = b \operatorname { s i n } ( \varphi ) \operatorname { c o s } ( \theta ) } \\
{ x _ { 2 } = b \operatorname { s i n } ( \varphi ) \operatorname { s i n } ( \theta ) } \\
{ x _ { 3 } = a \operatorname { c o s } ( \varphi ) }
\end{array} \quad \left\{\begin{array}{l}
\rho=b \sin (\varphi) \\
\theta=\theta \\
z=x_{3}=a \cos (\varphi)
\end{array}\right.\right.
$$

and

$$
\left\{\begin{array}{l}
\underline{e}_{\lambda}=\frac{1}{L_{\lambda}}\left\{a \sin (\varphi) \underline{e}_{\rho}+b \cos (\varphi) \underline{e}_{3}\right\} \\
\underline{e}_{\varphi}=\frac{1}{L_{\lambda}}\left\{b \cos (\varphi) \underline{e}_{\rho}-a \sin (\varphi) \underline{e}_{3}\right\} \\
\underline{e}_{\theta}=\underline{e}_{\theta}
\end{array}\right.
$$

with $L_{\lambda}=\sqrt{a^{2} \sin ^{2}(\varphi)+b^{2} \cos ^{2}(\varphi)}, \lambda \in\left[0,+\infty\left[, \varphi \in[0, \pi]\right.\right.$ and $\underline{e}_{\rho}=\cos (\theta) \underline{e}_{1}+$ $\sin (\theta) \underline{e}_{2}, \theta \in[0,2 \pi]$. In the above equations: $a=c \cosh (\lambda)$ and $b=c \sinh (\lambda)$ for a prolate void, while for the case of an oblate void $a=c \sinh (\lambda)$ and $b=$ $c \cosh (\lambda)$. The iso- $\lambda$ surfaces define confocal spheroids with foci $c=\sqrt{\left|a^{2}-b^{2}\right|}$ and eccentricity $e=c / a$, for a prolate void while $e=c / b$ for an oblate one. The porosity $f$ is defined by: $f=\left(a_{1} b_{1}^{2}\right) /\left(a_{2} b_{2}^{2}\right)$. The matrix of the spheroidal unit cell is made of a rigid-plastic material obeying to the von Mises yield criterion, $\sigma_{e q} \leq \sigma_{0}$, $\left(\sigma_{0}\right.$ being the yield stress in tension and $\sigma_{e q}$ the von Mises 
equivalent stress $\left.\sigma_{e q}=\sqrt{\frac{3}{2} \overline{\boldsymbol{\sigma}}: \overline{\boldsymbol{\sigma}}}\right)$ and the associated flow rule:

$$
\overline{\boldsymbol{\sigma}}=\frac{2 \sigma_{0}}{3} \frac{\boldsymbol{d}}{d_{e q}}, \quad \text { with } d_{e q}=\sqrt{\frac{2}{3} \boldsymbol{d}: \boldsymbol{d}}, \quad \boldsymbol{d}=\frac{1}{2}(\nabla \otimes \underline{v}+\underline{v} \otimes \nabla)
$$

where $\underline{v}$ is the velocity field, $\boldsymbol{d}$ the strain rate tensor and $d_{e q}$ the von Mises equivalent strain rate. $\overline{\boldsymbol{\sigma}}$ represents the deviatoric part of the local stress tensor.

The surface between the void and the solid matrix, denoted $\Gamma$, is assumed to be described by a stress-type interface model, which was introduced by [36] in the context of elasticity and extended by $[18,50]$ to plasticity. Stress interfaces ensure the continuity of the velocity field while the traction vector, $\underline{t}=\boldsymbol{\sigma} \cdot \underline{n}$, undergoes a jump which is governed by the Young-Laplace equation:

$$
[\underline{t}]_{\Gamma}=-\operatorname{div}_{s}(\boldsymbol{\tau})
$$

In the above equation, $\operatorname{div}_{s}(\boldsymbol{\tau})$ denotes the surface divergence of tensor $\boldsymbol{\tau}$ such that vector $\operatorname{div}_{s}(\boldsymbol{\tau})=(\boldsymbol{\tau} \otimes \underline{\nabla}): \boldsymbol{P}(\underline{n})$, with $\boldsymbol{P}(\underline{n})=\boldsymbol{I}-\underline{n} \otimes \underline{n}, \boldsymbol{I}$ being the second order identity tensor and $\underline{n}$ the normal unit vector taken on the interface $\Gamma$ and oriented from the void to the solid matrix. In (5), the notation $[\underline{t}]_{\Gamma}$ represents the jump of $\underline{t}$ across $\Gamma$ defined as follows: $[\underline{t}]_{\Gamma}=\underline{t}^{+}-\underline{t}^{-}$, where $\underline{t}^{+}$and $\underline{t}^{-}$are the values of the traction vector calculated on both sides of $\Gamma$. When the interface $\Gamma$ is the boundary between a void and a solid, $\underline{t}^{-}=0$ and relation (5) reduces to $\underline{t}^{+}=-\operatorname{div}_{s}(\boldsymbol{\tau})$.

In the Gurtin model [36], the interfacial stress $\boldsymbol{\tau}$ has two contributions, the first one is attributed to the presence of interfacial residual stresses, independent of the deformation, while the second one is related to elastic deformation of the interface. This model has been later extended in the context of plasticity by Monchiet and Bonnet [51] along a method formalized by Benveniste [4] in the context of linear elasticity. This method is based on the concept of equivalent elastic interphase which accounts for the presence of a few layers of atoms having a different local environment than atoms in the bulk. By considering the appropriate values for the elasticity coefficients of the interphase, it can be replaced by an idealized imperfect interface involving the jump of the traction vector which is described by the Gurtin model. Later, [51] have extended the work of [4] to the case of a plastic interphase. The interfacial model obtained in this work induces a jump of the traction vector which is still given by equation (5). However, in (5), the interfacial stress $\boldsymbol{\tau}$ is related to the plastic deformation of the interface. Its expression is:

$$
\boldsymbol{\tau}=\tau_{r} \boldsymbol{P}(\underline{n})+\frac{2 \tau_{0}}{3 d_{e q}^{s}}\left(\boldsymbol{d}_{s}+\operatorname{tr}\left(\boldsymbol{d}_{s}\right) \boldsymbol{P}(\underline{n})\right)
$$


where $\tau_{r}$ is the interfacial residual stress, $\tau_{0}$ is a material parameter. Although limited, the particular form $\tau_{r} \boldsymbol{P}(\underline{n})$ (isotropic in the plane of the interface), considered for the interfacial residual stress is able to represent some cases of practical interest such as capillary forces. Note that recent ab initio based calculations by [71] indicate a slight anisotropy for these residual stresses.

In $(6), \boldsymbol{d}_{s}$ is the interfacial plastic strain rate which is defined as the restriction of the total strain rate to the tangent plane of normal unit vector $\underline{n}$. In (6), $d_{e q}^{s}$ is the surface equivalent strain rate. The expressions of $\boldsymbol{d}_{s}$ and $d_{e q}^{s}$ are:

$$
\begin{aligned}
& \boldsymbol{d}_{s}=\boldsymbol{P}(\underline{n}) \cdot \boldsymbol{d} \cdot \boldsymbol{P}(\underline{n}) \\
& d_{e q}^{s}=\left[\frac{2}{3}\left(\boldsymbol{d}_{s}: \boldsymbol{d}_{s}+\left(\operatorname{tr} \boldsymbol{d}_{s}\right)^{2}\right)\right]^{1 / 2}
\end{aligned}
$$

Note that for a spheroidal surface $\underline{n}=\underline{e}_{\lambda}, \boldsymbol{P}(\underline{n})=\underline{e}_{\theta} \otimes \underline{e}_{\theta}+\underline{e}_{\varphi} \otimes \underline{e}_{\varphi}$ and the quantities $\boldsymbol{d}_{s}$ and $d_{e q}^{s}$ read:

$$
\begin{aligned}
& \boldsymbol{d}_{s}=d_{\theta \theta} \underline{e}_{\theta} \otimes \underline{e}_{\theta}+d_{\varphi \varphi} \underline{e}_{\varphi} \otimes \underline{e}_{\varphi}+d_{\theta \varphi}\left(\underline{e}_{\theta} \otimes \underline{e}_{\varphi}+\underline{e}_{\varphi} \otimes \underline{e}_{\theta}\right) \\
& d_{e q}^{s}=\left[\frac{4}{3}\left(d_{\theta \theta}^{2}+d_{\varphi \varphi}^{2}+d_{\theta \varphi}^{2}+d_{\theta \theta} d_{\varphi \varphi}\right)\right]^{1 / 2}
\end{aligned}
$$

\subsection{Limit analysis taking into consideration interfacial stresses}

As already mentioned, Hill-Mandel kinematic homogenization approach will be used to derive the overall plastic potential of the porous solid. Thus, uniform strain rate boundary conditions are considered on external surface of the hollow spheroid:

$$
\underline{v}\left(\lambda=\lambda_{2}\right)=\boldsymbol{D} \cdot \underline{x}
$$

where $\boldsymbol{D}$ is the macroscopic strain rate tensor. 


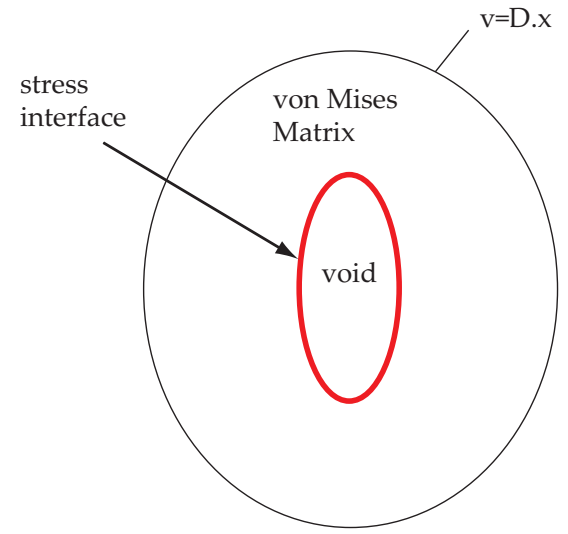

Fig. 2. Schematic showing the hollow spheroid with a stress interface.

The limit stress at the macroscopic scale, $\boldsymbol{\Sigma}$, is:

$$
\boldsymbol{\Sigma}=\frac{\partial \Pi}{\partial \boldsymbol{D}}(\boldsymbol{D})
$$

where $\Pi(\boldsymbol{D})$, is the macroscopic dissipation given by:

$$
\Pi(\boldsymbol{D})=\inf _{\underline{v} \in \mathcal{K}} \frac{1}{V_{2}}\left[\int_{\Omega-\omega} \sigma_{0} d_{e q} d V+\int_{\Gamma}[\underline{t}]_{\Gamma} \cdot \underline{v} d S\right]
$$

where the infimum is taken on $\mathcal{K}$ which is the space of admissible velocity fields, i.e. of continuous and differentiable velocity fields that comply with condition (9). The first integral in (11) is computed over the solid matrix, defined by $\Omega-\omega$, where $\Omega$ is the domain corresponding to the unit cell, while $\omega$ is the domain occupied by the void. The second integral in (11) is performed over the surface $\Gamma$ of the void. It is recalled that $V_{2}=4 \pi a_{2} b_{2}^{2} / 3$ is the volume of $\Omega$. Using the generalized Young-Laplace equation (5), it is readily seen that the second integral is related to the interfacial residual stress and interfacial plastic strain. It then follows that:

$$
\int_{\Gamma}[]_{\Gamma} \cdot \underline{v} d S=-\int_{\Gamma} \operatorname{div}_{s}(\boldsymbol{\tau}) \cdot \underline{v} d S
$$

Moreover, for any continuously differentiable two order tensor $\boldsymbol{\tau}$ and vector $\underline{v}$,

$$
\operatorname{div}_{s}(\boldsymbol{\tau} \cdot \underline{v})=\operatorname{div}_{s}(\boldsymbol{\tau}) \cdot \underline{v}+\boldsymbol{\tau}: \boldsymbol{d}_{s}
$$

The integral over $\Gamma$ of the quantity $\operatorname{div}_{s}(\boldsymbol{\tau} . \underline{v})$ being null over any closed surface, it follows that: 


$$
\int_{\Gamma} \operatorname{div}_{s}(\boldsymbol{\tau}) \cdot \underline{v} d S=-\int_{\Gamma} \boldsymbol{\tau}: \boldsymbol{d}_{s} d S
$$

Finally, replacing $\boldsymbol{\tau}$ by its expression (6), one obtains:

$$
\Pi(\boldsymbol{D})=\inf \left[\frac{1}{V_{2}} \int_{\Omega-\omega} \sigma_{0} d_{e q} d V+\frac{1}{V_{2}} \int_{\Gamma} \tau_{0} d_{e q}^{s} d S+\frac{1}{V_{2}} \int_{\Gamma} \tau_{r} \operatorname{tr}\left(\boldsymbol{d}^{s}\right) d S\right]
$$

Note that this expression of $\Pi(\boldsymbol{D})$ contains three terms: the first is classic in the context of limit analysis of ductile porous media; the last two terms are associated to interfacial plastic dissipations.

Note that, in the spheroidal coordinate system, the surface integral is defined by:

$$
\int_{\Gamma} \bullet d S=\int_{\varphi=0}^{\varphi=\pi} \int_{\theta=0}^{\theta=2 \pi} \bullet b_{1} L_{\lambda_{1}} \sin (\varphi) d \varphi d \theta
$$

where $L_{\lambda_{1}}$ is the value of $L_{\lambda}$ for $\lambda=\lambda_{1}$, i.e. $L_{\lambda_{1}}=\sqrt{a_{1}^{2} \sin ^{2}(\varphi)+b_{1}^{2} \cos ^{2}(\varphi)}$; for the definition of the volume integral in the expression of $\Pi(\boldsymbol{D})$, the reader is referred to $[31,32]$.

\section{The choice of the trial velocity fields}

A crucial step in the derivation of an approximate closed form expression of the macroscopic potential of the ductile porous material lies in the choice of the trial velocity field. Generally, this field is composed in two parts: one involving a constant traceless tensor $\boldsymbol{A}$, one, denoted $\underline{v}^{E}$, which is heterogeneous, i.e.

$$
\underline{v}=\boldsymbol{A} \cdot \underline{x}+B \underline{v}^{E}
$$

We propose here to adopt, for $\underline{v}^{E}$, the velocity field considered by Gologanu et al. [31,32] and Monchiet et al. [49]. This field has the property to comply with uniform strain rate conditions on any iso- $\lambda=c s t$ spheroid. Gologanu et al. [33], Monchiet et al. [51] considered more sophisticated trial velocity fields in order to derive an improved estimate of the macroscopic yield locus of ductile porous media. In these approaches, the trial velocity field introduce a larger number of parameters which cannot be entirely determined by the boundary conditions. Due to additional difficulties related to the consideration of interfacial stress effects, we propose to restrict our analysis with the trial velocity fields first considered in $[31,32]$. Its expression is: 


$$
\left\{\begin{array}{l}
v_{\lambda}^{E}=\frac{c^{3}}{b L_{\lambda}}\left[1+\frac{1}{2}(1-3 \alpha)\left(1-3 \cos ^{2}(\varphi)\right)\right] \\
v_{\varphi}^{E}=\frac{3 c^{3}}{4 a b^{2} L_{\lambda}}\left[(1-\alpha) b^{2}-2 \alpha a^{2}\right] \sin (2 \varphi) \\
v_{\theta}^{E}=0
\end{array}\right.
$$

In which $\alpha$ depends on $\lambda$ or equivalently $e$ and is given by:

$$
\alpha= \begin{cases}\frac{a b^{2}}{c^{3}} \operatorname{arctanh}\left\{\frac{c}{a}\right\}-\frac{b^{2}}{c^{2}}=\frac{1-e^{2}}{e^{3}}(\operatorname{arctanh}(e)-e) & \text { (prolate void) } \\ -\frac{a b^{2}}{c^{3}} \arctan \left\{\frac{c}{a}\right\}+\frac{b^{2}}{c^{2}}=\frac{e-\arcsin (e) \sqrt{1-e^{2}}}{e^{3}} & \text { (oblate void) }\end{cases}
$$

For the explicit dependence of $a$ and $b$ on the coordinates $\lambda$ and $e$, the reader is referred to section 2.1. The verification of uniform strain rate boundary

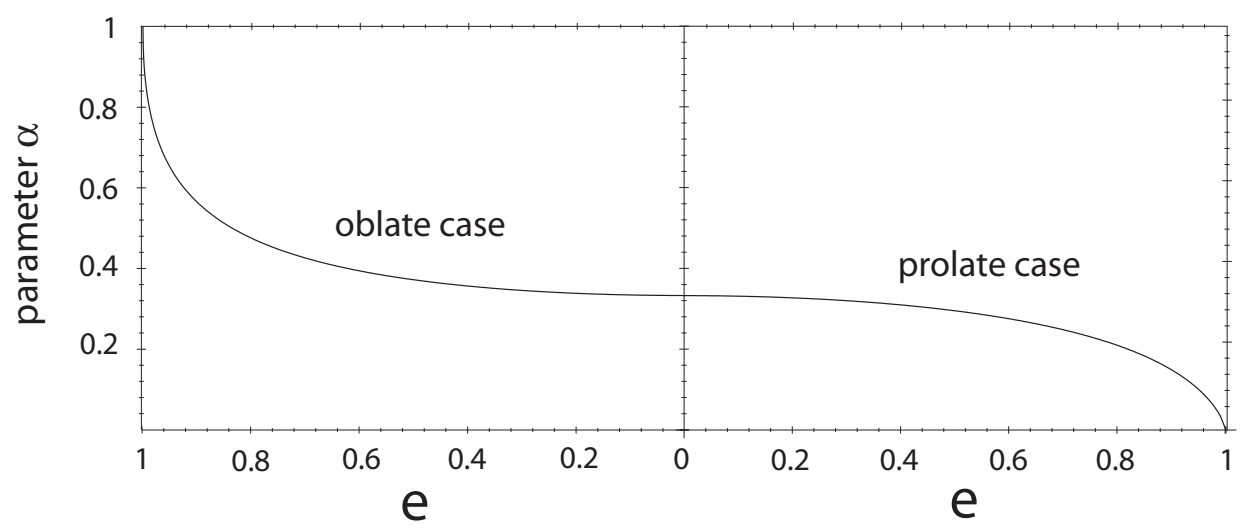

Fig. 3. $\alpha$ as function of $e$ for a prolate and an oblate void.

conditions at the outer spheroid $\lambda=\lambda_{2}$ leads to:

$$
\boldsymbol{A}=\boldsymbol{D}-D_{m} \boldsymbol{T}_{2} ; \quad B=\frac{a_{2} b_{2}^{2}}{c^{3}} D_{m}
$$

where $\boldsymbol{T}_{2}=\boldsymbol{T}\left(\lambda_{2}\right)$, with

$$
\boldsymbol{T}(\lambda)=3 \frac{a_{2} b_{2}^{2}}{a b^{2}}\left[\frac{1}{2}(1-\alpha) \boldsymbol{I}_{t}+\alpha \boldsymbol{I}_{n}\right]
$$

and

$$
\boldsymbol{I}_{t}=\underline{e}_{1} \otimes \underline{e}_{1}+\underline{e}_{2} \otimes \underline{e}_{2}, \quad \boldsymbol{I}_{n}=\underline{e}_{3} \otimes \underline{e}_{3}
$$


These tensors are the two projectors associated to the transverse isotropy related to the $O x_{3}$ axis of the spheroidal cavity. They comply to: $\boldsymbol{I}_{n} \cdot \boldsymbol{I}_{n}=\boldsymbol{I}_{n}$, $\boldsymbol{I}_{t} \cdot \boldsymbol{I}_{t}=\boldsymbol{I}_{t}, \boldsymbol{I}_{n} . \boldsymbol{I}_{t}=0$ and $\boldsymbol{I}=\boldsymbol{I}_{n}+\boldsymbol{I}_{t}$ where is recalled that $\boldsymbol{I}$ is the second order identity tensor. From equation (18), it is readily seen that the strain rate field in the matrix is the sum of a homogenous deviatoric field $\boldsymbol{A}$ and a non-homogeneous field $\boldsymbol{d}^{E}$ :

$$
\boldsymbol{d}=\boldsymbol{A}+B \boldsymbol{d}^{E}
$$

with $\boldsymbol{d}^{E}$ given by:

$$
\left\{\begin{array}{l}
d_{\lambda \lambda}^{E}=-\frac{3 c^{3}}{a b^{2}}(1-\alpha)+\frac{3 c^{3} a}{2 b^{2} L_{\lambda}^{2}}(1-3 \alpha) \sin ^{2}(\varphi) \\
d_{\varphi \varphi}^{E}=\frac{3 c^{3}}{2 a b^{2}}(1-\alpha)-\frac{3 c^{3} a}{2 b^{2} L_{\lambda}^{2}}(1-3 \alpha) \sin ^{2}(\varphi) \\
d_{\theta \theta}^{E}=\frac{3 c^{3}}{2 a b^{2}}(1-\alpha) \\
d_{\lambda \varphi}^{E}=\frac{3 c^{3}}{4 b L_{\lambda}^{2}}(1-3 \alpha) \sin (2 \varphi)
\end{array}\right.
$$

Note that the strain field $\boldsymbol{d}^{E}$ can be put into the form the compact form:

$$
\boldsymbol{d}^{E}=\frac{c^{3}}{a_{2} b_{2}^{2}}\left[\boldsymbol{T}(\lambda)-3 \frac{a_{2} b_{2}^{2}}{a b^{2}} \underline{e}_{\lambda} \otimes \underline{e}_{\lambda}\right]
$$

In relation (23), $\boldsymbol{A}$ and $B$ are replaced by expressions (20) while relation (25) is used for $\boldsymbol{d}^{E}$. The total strain rate, $\boldsymbol{d}$, reads then:

$$
\boldsymbol{d}=\boldsymbol{D}+D_{m}\left[\boldsymbol{T}(\lambda)-\boldsymbol{T}_{2}-3 \frac{a_{2} b_{2}^{2}}{a b^{2}} \underline{e}_{\lambda} \otimes \underline{e}_{\lambda}\right]
$$

where it is recalled that $\boldsymbol{T}_{2}=\boldsymbol{T}\left(\lambda_{2}\right)$ and $\boldsymbol{T}(\lambda)$ is given by (21). Taking into account this expression, the surface strain rate tensor, $\boldsymbol{d}_{s}$, defined by the first relation in (7) reads:

$$
\boldsymbol{d}_{s}=\boldsymbol{P}(\theta, \varphi) \cdot\left[\boldsymbol{D}+D_{m}\left(\boldsymbol{T}_{1}-\boldsymbol{T}_{2}\right)\right] \cdot \boldsymbol{P}(\theta, \varphi)
$$

for which account has been taken of the identity $\boldsymbol{P}(\varphi, \theta) \cdot \underline{e}_{\lambda}=0$ and $\boldsymbol{T}_{1}=$ $\boldsymbol{T}\left(\lambda_{1}\right)$. As it will be shown in the next section, the representation of the interfacial plastic strain rate tensor, in the form given by $(27)$, is required for the 
computation of the integrals which enter into the definition of $\Pi(\boldsymbol{D})$ given by (11).

\section{Closed-form expression of the macroscopic dissipation}

The trial velocity field being entirely determined by the boundary conditions, no minimization procedure is required for $\Pi(\boldsymbol{D})$ which, according to (15), can be put into the form:

$$
\Pi(\boldsymbol{D})=\Pi_{1}(\boldsymbol{D})+\Pi_{2}(\boldsymbol{D})+\Pi_{3}(\boldsymbol{D})
$$

where the quantities $\Pi_{1}(\boldsymbol{D}), \Pi_{2}(\boldsymbol{D})$ and $\Pi_{3}(\boldsymbol{D})$ read:

$$
\begin{aligned}
& \Pi_{1}(\boldsymbol{D})=\frac{1}{V_{2}} \int_{\Omega-\omega} \sigma_{0} d_{e q} d V \\
& \Pi_{2}(\boldsymbol{D})=\frac{1}{V_{2}} \int_{\Gamma} \tau_{r} \operatorname{tr}\left(\boldsymbol{d}_{s}\right) d S \\
& \Pi_{3}(\boldsymbol{D})=\frac{1}{V_{2}} \int_{\Gamma} \tau_{0} d_{e q}^{s} d S
\end{aligned}
$$

Expressions of these quantities will be provided in the following sections, 4.1, 4.2 and 4.3 , respectively.

\subsection{Approximate expression of $\Pi_{1}(\boldsymbol{D})$}

Based on the trial velocity field (17) (together with (18)), Gologanu et al. $[31,32]$ derived closed-form expressions of $\Pi_{1}(\boldsymbol{D})$ and then a two-field estimate of the macroscopic criterion in the cases of a prolate and an oblate cavity. Although the two approximate expressions obtained by these authors prove to be accurate, comparatively to the exact two-field solution (evaluated numerically without any approximations), we propose to use the unified expression established in [49], valid for both cases of a prolate and an oblate cavity. The latter, derived in the more general case of an anisotropic Hill plastic porous medium with spheroidal cavities, reduced in the limit case of the von Mises matrix to:

$$
\Pi_{1}(\boldsymbol{D})=\sigma_{0} f\left[Y \operatorname{arcsinh}\left\{\frac{u Y}{X}\right\}-\frac{\sqrt{X^{2}+u^{2} Y^{2}}}{u}\right]_{u=u_{1}}^{u=u_{2}}
$$


in which the quantities $X$ and $Y$ depend on the macroscopic strain rate tensor as:

$$
\begin{aligned}
& X=\left[(1-\zeta)\left(D_{q}+\left(1-3 \alpha_{2}\right) D_{m}\right)^{2}+D_{s}^{2}+D_{t}^{2}\right]^{1 / 2}, \\
& Y=\frac{1}{f \kappa}\left[3+\eta\left(1-3 \alpha_{2}\right)\right] D_{m}+\frac{\eta}{f \kappa} D_{q}
\end{aligned}
$$

Expressions of coefficients $\eta, \kappa$ and $\zeta$ as well as $u_{1}$ and $u_{2}$ are reported in appendix A. The invariant quantities, $D_{q}, D_{s}$ and $D_{t}$, in agreement with the void-induced transverse isotropy (with a symmetry axis $O x_{3}$ ), are defined as:

$$
\left\{\begin{array}{l}
D_{q}=\frac{2}{3} D_{33}-\frac{1}{3}\left(D_{11}+D_{22}\right) \\
D_{s}=\frac{1}{\sqrt{3}} \sqrt{\left(D_{22}-D_{11}\right)^{2}+4 D_{12}^{2}} \\
D_{t}=\frac{2}{\sqrt{3}} \sqrt{D_{13}^{2}+D_{23}^{2}}
\end{array}\right.
$$

The macroscopic equivalent strain rate can then be decomposed as $D_{e q}^{2}=$ $D_{q}^{2}+D_{s}^{2}+D_{t}^{2}$.

\subsection{Exact expression for $\Pi_{2}(\boldsymbol{D})$}

We now propose to compute the exact expression of the dissipation $\Pi_{2}(\boldsymbol{D})$ associated to the interfacial residual stresses. Introducing expression (27) in the second relation in (29) leads to:

$$
\Pi_{2}(\boldsymbol{D})=\frac{\tau_{r} S_{1}}{V_{2}}\left[\boldsymbol{D}+D_{m}\left(\boldsymbol{T}_{1}-\boldsymbol{T}_{2}\right)\right]: \overline{\boldsymbol{P}}
$$

where the second order tensor $\overline{\boldsymbol{P}}$ is defined by:

$$
\overline{\boldsymbol{P}}=\frac{1}{S_{1}} \int_{\Gamma} \boldsymbol{P}(\theta, \varphi) d S=\frac{1}{2}(1+\gamma) \boldsymbol{I}_{t}+(1-\gamma) \boldsymbol{I}_{n}
$$

and $S_{1}$ is the area of the spheroid of coordinate $\lambda=\lambda_{1}$ :

$$
S_{1}= \begin{cases}\frac{2 \pi a_{1}^{2} b_{1}}{c} \arctan \left\{\frac{c}{b_{1}}\right\}+2 \pi b_{1}^{2} & \text { (prolate void) } \\ \frac{2 \pi a_{1}^{2} b_{1}}{c} \operatorname{arctanh}\left\{\frac{c}{b_{1}}\right\}+2 \pi b_{1}^{2} & \text { (oblate void) }\end{cases}
$$


The parameter $\gamma$, which depends on the cavity shape, reads:

$$
\gamma=\frac{b_{1}^{2}}{a_{1}^{2}-b_{1}^{2}}\left(1-\frac{4 b_{1}^{2} \pi}{S_{1}}\right)
$$

For more details concerning the computation of the integral in (34), one may refer to appendix B.

The value of $\gamma$ for the particular cases of a spherical, cylindrical and pennyshaped crack are provided in table 1 . The variation of $\gamma$ with the void eccentricity $e_{1}$ are given on figure 4 . It can be noted that, for any value of eccentricity, $\gamma$ lies in the range $[0,1]$.

Table 1

\begin{tabular}{c|ccc}
\hline & sphere & cylinder & crack \\
\hline$\gamma$ & $1 / 3$ & 0 & 1 \\
\hline
\end{tabular}

Values of $\gamma$ for some particular cases of a spheroidal cavity.

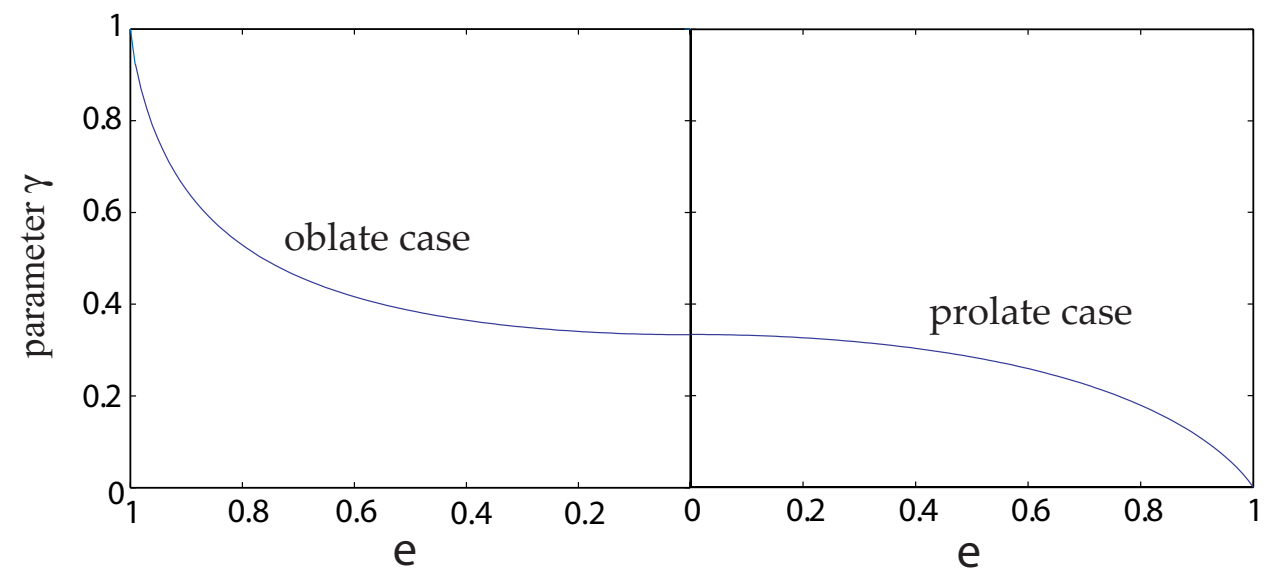

Fig. 4. $\gamma$ as function of $e_{1}$ for a prolate and an oblate void.

Interestingly, $\Pi_{2}(\boldsymbol{D})$, given by (33), can be put into the form:

$$
\Pi_{2}(\boldsymbol{D})=\frac{\tau_{r} S_{1}}{V_{1}} \boldsymbol{D}: \boldsymbol{N}
$$

where $V_{1}=4 \pi a_{1} b_{1}^{2} / 3$ is the volume of the spheroidal cavity while the second order tensor $\boldsymbol{N}$ is given by:

$$
\boldsymbol{N}=\left[\mathbb{I}+\frac{1}{3} \boldsymbol{I} \otimes\left(\boldsymbol{T}_{1}-\boldsymbol{T}_{2}\right)\right]: \overline{\boldsymbol{P}}
$$


$\mathbb{I}$, whose components are $I_{i j k l}=\left(\delta_{i k} \delta_{j l}+\delta_{i l} \delta_{j k}\right) / 2$, is the fourth order identity tensor, $\delta_{i j}$ being the Kronecker symbol. From the definition (21) of $\boldsymbol{T}(\lambda)$ and the expression (34) of $\overline{\boldsymbol{P}}$, one obtains:

$$
\begin{aligned}
\boldsymbol{N}= & \frac{1}{2}\left[1+\gamma+\left(\alpha_{1}-f \alpha_{2}\right)(1-3 \gamma)\right] \boldsymbol{I}_{t} \\
& +\left[1-\gamma-\frac{1}{2}\left(1-\alpha_{1}-f\left(1-\alpha_{2}\right)(1-3 \gamma)\right)\right] \boldsymbol{I}_{n}
\end{aligned}
$$

This tensor depends on the eccentricity $e_{1}, e_{2}$ (through $\alpha_{1}, \alpha_{2}$ and $\gamma$ in (19) and (36)) and of the porosity $f$. In the particular case of spherical void, $\boldsymbol{N}$ reduces to $\frac{2}{3} \boldsymbol{I}$. In the general case, it is convenient to express $\Pi_{2}(\boldsymbol{D})$ as function of $D_{m}, D_{q}, D_{s}$ and $D_{t}$, given in (32):

$$
\Pi_{2}(\boldsymbol{D})=\frac{\tau_{r} S_{1}}{V_{1}}\left(g_{1} D_{m}+g_{2} D_{q}\right)
$$

with:

$$
g_{1}=2-\frac{1}{2}(1-3 \gamma)\left(1-3 \alpha_{1}-f\left(1-3 \alpha_{2}\right)\right), \quad g_{2}=\frac{f}{2}(1-3 \gamma)
$$

\subsection{An upper bound for $\Pi_{3}(\boldsymbol{D})$}

We are now interested by the derivation of the dissipation $\Pi_{3}(\boldsymbol{D})$ associated to the plastic deformation of the surface $\Gamma$. It is defined by the last relation in (29), in which the interfacial equivalent strain rate, $d_{e q}^{s}$, is given by (7). The required expressions, $(7)$ and $(27)$, for the interfacial plastic strain rate leads to:

$$
d_{e q}^{s}=\left[\frac{2}{3}\left(\boldsymbol{D}+D_{m}\left(\boldsymbol{T}_{1}-\boldsymbol{T}_{2}\right)\right): \mathbb{P}(\theta, \varphi):\left(\boldsymbol{D}+D_{m}\left(\boldsymbol{T}_{1}-\boldsymbol{T}_{2}\right)\right)\right]^{1 / 2}
$$

where the fourth order tensor $\mathbb{P}(\theta, \varphi)$ is given by:

$$
\mathbb{P}(\theta, \varphi)=\boldsymbol{P}(\theta, \varphi) \underline{\bar{\otimes}} \boldsymbol{P}(\theta, \varphi)+\boldsymbol{P}(\theta, \varphi) \otimes \boldsymbol{P}(\theta, \varphi)
$$

for which the following notation has been used (for any couple of second order tensors $\boldsymbol{a}$ and $\boldsymbol{b}$ ):

$$
(\boldsymbol{a} \underline{\bar{\otimes} \boldsymbol{b}})_{i j k l}=\frac{1}{2}\left(a_{i k} b_{j l}+a_{i l} b_{j k}\right)
$$


At this stage, it is not possible to derive a closed-form expression of $\Pi_{3}(\boldsymbol{D})$, even in the simplest case of a spherical void (see [18]). However, based on the Cauchy-Schwartz inequality, one can establish an upper bound for $\Pi_{3}(\boldsymbol{D})$. Indeed, for the positive and square integrable function $d_{e q}^{s}$, one has:

$$
\frac{1}{S_{1}} \int_{\Gamma} d_{e q}^{s} d S \leq \sqrt{\frac{1}{S_{1}} \int_{\Gamma}\left(d_{e q}^{s}\right)^{2} d S}
$$

The following approximation is then used:

Approximation $\mathcal{A} 1$ : In the expression of $\Pi_{3}(\boldsymbol{D})$ the integral of the interfacial equivalent strain rate over the surface of the spheroidal cavity, i.e. $\int_{\Gamma} d_{e q}^{s} d S$, is replaced by $S_{1}\left[\frac{1}{S_{1}} \int_{\Gamma}\left(d_{e q}^{s}\right)^{2} d S\right]^{1 / 2}$.

With this approximation, an upper bound of the plastic dissipation $\Pi_{3}(\boldsymbol{D})$ is:

$$
\begin{aligned}
\Pi_{3}(\boldsymbol{d}) & =\frac{S_{1}}{V_{2}}\left[\frac{1}{S_{1}} \int_{\Gamma}\left(d_{e q}^{s}\right)^{2} d S\right]^{1 / 2} \\
& =\frac{S_{1}}{V_{2}}\left[\frac{2}{3}\left(\boldsymbol{D}+D_{m}\left(\boldsymbol{T}_{1}-\boldsymbol{T}_{2}\right)\right): \overline{\mathbb{P}}:\left(\boldsymbol{D}+D_{m}\left(\boldsymbol{T}_{1}-\boldsymbol{T}_{2}\right)\right)\right]^{1 / 2}
\end{aligned}
$$

where the fourth order tensor $\overline{\mathbb{P}}$ is defined by:

$$
\begin{aligned}
\overline{\mathbb{P}}= & \frac{1}{S_{1}} \int_{\Gamma} \mathbb{P}(\theta, \varphi) d S=(3 \gamma+\mu) \mathbb{E}_{1}+2 \mu \mathbb{E}_{2}+\frac{2 \gamma+\mu}{2} \mathbb{E}_{3} \\
& +\left[\frac{5}{2}(1-\gamma)-2 \mu\right] \mathbb{E}_{4}+\left[\frac{3}{2}(1-\gamma)-\mu\right]\left(\mathbb{E}_{5}+\mathbb{E}_{6}\right)
\end{aligned}
$$

$\mathbb{E}_{n}$ for $n=1 . .6$ are the fourth order tensors of the Walpole basis [65] (the elements of this basis are recalled in appendix B). Parameter $\mu$ is given by:

$$
\mu=\frac{a^{2}}{a^{2}-b^{2}}(1-3 \gamma)
$$

The details concerning the computation of the integral in (47) can be found in appendix B.

The values of $\mu$ for a spherical or a cylindrical void, as well as for a pennyshaped crack are provided in table 2 . For any value of the eccentricity, coefficient $\mu$ lies in the range $[0,1]$. Its variation with respect to $e_{1}$ is shown on figure 5 . 
Table 2

Values of $\mu$ for particular cases of a spheroidal cavity.

\begin{tabular}{c|ccc}
\hline & sphere & cylinder & crack \\
\hline$\mu$ & $8 / 15$ & 1 & 0 \\
\hline
\end{tabular}

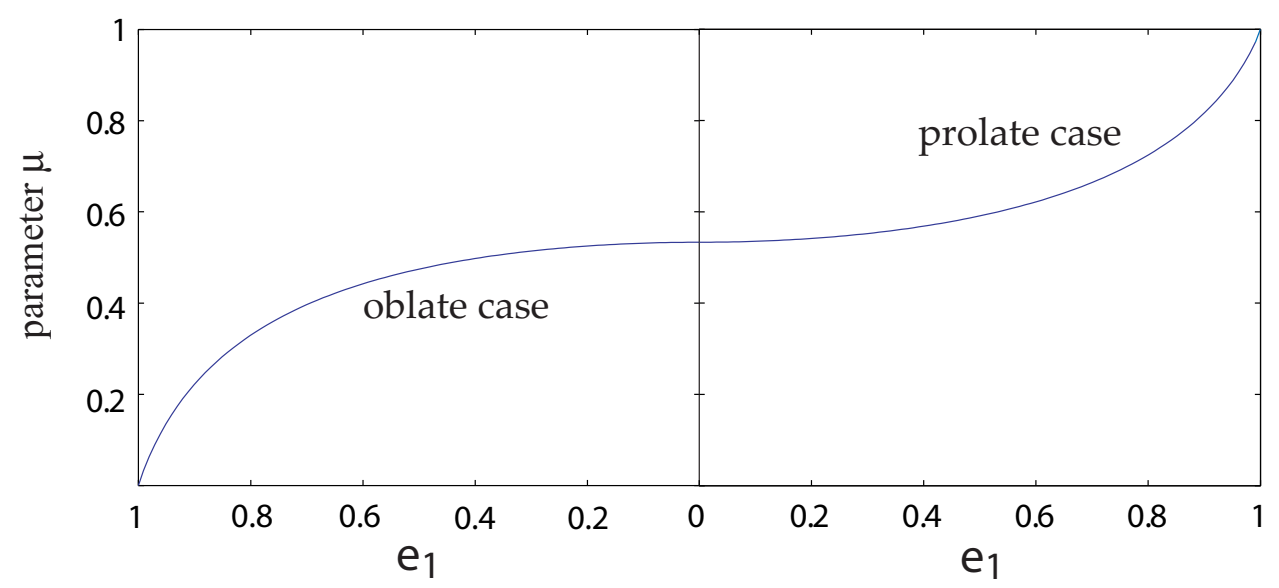

Fig. 5. Variations of $\mu$ with the eccentricity $e_{1}$ in the case of prolate and an oblate void.

Finally, $\Pi_{3}(\boldsymbol{D})$ can be put into the form:

$$
\Pi_{3}(\boldsymbol{D})=\frac{\tau_{0} S_{1}}{V_{1}} \sqrt{\frac{2}{3} \boldsymbol{D}: \mathbb{S}: \boldsymbol{D}}
$$

where the fourth order tensor $\mathbb{S}$ is defined by:

$$
\mathbb{S}=f^{2}\left[\mathbb{I}+\boldsymbol{I} \otimes\left(\boldsymbol{T}_{1}-\boldsymbol{T}_{2}\right)\right]: \overline{\mathbb{P}}:\left[\mathbb{I}+\left(\boldsymbol{T}_{1}-\boldsymbol{T}_{2}\right) \otimes \boldsymbol{I}\right]
$$

and the components of $\mathbb{S}$ are provided in appendix B. As already done for $\Pi_{1}(\boldsymbol{D})$ and $\Pi_{2}(\boldsymbol{D})$, we now express $\Pi_{3}(\boldsymbol{D})$ as function of the strain rate invariants introduced in (32):

$$
\Pi_{3}(\boldsymbol{D})=\frac{\tau_{0} S_{1}}{V_{1}} \sqrt{h_{1} D_{m}^{2}+h_{2} D_{q}^{2}+2 h_{3} D_{m} D_{q}+h_{4} D_{s}^{2}+h_{5} D_{t}^{2}}
$$

where the coefficients $h_{n}$ are given by: 


$$
\left\{\begin{array}{l}
h_{1}=(3 \gamma+3 \mu-2) U^{2}+2(3 \gamma-1) U+4 \\
h_{2}=f^{2}(3 \gamma+3 \mu-2) \\
h_{3}=f(3 \gamma+3 \mu-2) U+f(3 \gamma-1) \\
h_{4}=\frac{f^{2}}{2}(2 \gamma+\mu) \\
h_{5}=\frac{f^{2}}{2}(5-4 \mu-5 \gamma)
\end{array}\right.
$$

with $U=1-3 \alpha_{1}-f\left(1-3 \alpha_{2}\right)$, $\gamma$ being given by (36) and $\mu$ by (48).

\section{An estimate of the macroscopic yield surface}

\subsection{The parametric equations}

The macroscopic yield surface of the nanoporous medium is obtained from equation (10) in which the macroscopic dissipation $\Pi(\boldsymbol{D})$ is obtained as the sum of $\Pi_{1}(\boldsymbol{D}), \Pi_{2}(\boldsymbol{D})$ and $\Pi_{3}(\boldsymbol{D})$ whose expressions are given by (30), (40) and (51) respectively.

Equation (10) defines a hyper-surface of dimension 5 in the space of macroscopic stress. Note that $\Pi_{1}(\boldsymbol{D}), \Pi_{2}(\boldsymbol{D})$ and $\Pi_{3}(\boldsymbol{D})$ and then $\Pi(\boldsymbol{D})$ depends on the macroscopic strain rate tensor, $\boldsymbol{D}$, only through the invariants $D_{m}, D_{q}, D_{s}$ and $D_{t}$. The yield surface can then be deduced from the following parametric equations:

$$
\left\{\begin{array}{l}
\Sigma_{m}=\frac{1}{3} \frac{\partial \Pi}{\partial D_{m}} \\
\Sigma_{q}=\frac{\partial \Pi}{\partial D_{q}} \\
\Sigma_{s}=\frac{\partial \Pi}{\partial D_{s}} \\
\Sigma_{t}=\frac{\partial \Pi}{\partial D_{t}}
\end{array}\right.
$$

where $\Sigma_{m}, \Sigma_{q}, \Sigma_{s}$ and $\Sigma_{t}$ are the invariants of the macroscopic stress defined by: 


$$
\left\{\begin{array}{l}
\Sigma_{m}=\frac{1}{3}\left(\Sigma_{11}+\Sigma_{22}+\Sigma_{33}\right) \\
\Sigma_{q}=\Sigma_{33}-\frac{1}{2}\left(\Sigma_{11}+\Sigma_{22}\right) \\
\Sigma_{s}=\frac{\sqrt{3}}{2} \sqrt{\left(\Sigma_{22}-\Sigma_{11}\right)^{2}+4 \Sigma_{12}^{2}} \\
\Sigma_{t}=\sqrt{3} \sqrt{\Sigma_{13}^{2}+\Sigma_{23}^{2}}
\end{array}\right.
$$

Relations (53) corresponds to a surface of dimension 3 in the space of dimension 4 defined by $\left(\Sigma_{m}, \Sigma_{q}, \Sigma_{s}, \Sigma_{t}\right)$. Unfortunately, for general loading case, it is not possible to derive a closed-form solution of the system given by (53), even for cylindrical or spherical voids. However, we succeed to identify closed-form expressions in some particular cases of macroscopic stress loading or values of the material parameters $\sigma_{0}, \tau_{0}$ and $\tau_{r}$. These solutions are presented in the next section.

The impossibility to obtain an explicit expression of the macroscopic yield surface does not matter by itself since relations (53) provide a set of parametric equations which are sufficient for the representation of the yield surface. Introducing the following three non dimensional variables $\xi_{1}=D_{q} / D_{m}$, $\xi_{2}=D_{s} / D_{m}$ and $\xi_{3}=D_{t} / D_{m}$, relations (53) can be rewritten as:

$$
\left\{\begin{array}{l}
\Sigma_{m}=\mathcal{G}_{1}\left(\xi_{1}, \xi_{2}, \xi_{3}\right) \\
\Sigma_{q}=\mathcal{G}_{2}\left(\xi_{1}, \xi_{2}, \xi_{3}\right) \\
\Sigma_{s}=\mathcal{G}_{3}\left(\xi_{1}, \xi_{2}, \xi_{3}\right) \\
\Sigma_{t}=\mathcal{G}_{4}\left(\xi_{1}, \xi_{2}, \xi_{3}\right)
\end{array}\right.
$$

Functions $\mathcal{G}_{n}\left(\xi_{1}, \xi_{2}, \xi_{3}\right)$ for $n=1,2,3,4$ are explicitly given in appendix C. The macroscopic yield surface can then be obtained by varying $\xi_{1}, \xi_{2}$ and $\xi_{3}$ from $-\infty$ to $+\infty$.

Remark 1: The term corresponding to $\Pi_{2}(\boldsymbol{D})$ is linear with respect to the macroscopic strain rate tensor, $\boldsymbol{D}$; this introduces the constant tensor $\frac{\tau_{r} S_{1}}{V_{1}} \boldsymbol{N}$ in equation (10). The derivatives of $\Pi_{1}(\boldsymbol{D})$ and $\Pi_{3}(\boldsymbol{D})$ according to $\boldsymbol{D}$ are null for $\boldsymbol{D}=0$. Consequently, the interfacial residual stress, through the term $\frac{\tau_{r} S_{1}}{V_{1}} \boldsymbol{N}$, induces a change in position of the center of the macroscopic yield surface. Since tensor $\boldsymbol{N}$ is axisymmetric (it has only two components along $\boldsymbol{I}_{t}$ and $\boldsymbol{I}_{n}$ ), the residual stress translates the macroscopic yield locus along the direction related to the macroscopic stress defined by $\Sigma_{m}$ and $\Sigma_{q}$. The position of the center in the space $\left(\Sigma_{m}, \Sigma_{q}, \Sigma_{s}, \Sigma_{t}\right)$ is denoted $\left(C_{m}, C_{q}, 0,0\right)$ where $C_{m}$ 
and $C_{q}$ are given by:

$$
C_{m}=\frac{\tau_{r} S_{1}}{3 V_{1}} g_{1}, \quad C_{q}=\frac{\tau_{r} S_{1}}{V_{1}} g_{2}
$$

Coefficients $g_{1}$ and $g_{2}$ are given by relations (41). Note also that $C_{q}$ is null in the case of a spherical void (corresponding to $\gamma=1 / 3$ ) which suggests that the interfacial residual stress $\tau_{r}$ only induces a translation of the macroscopic yield locus along the axis related to $\Sigma_{m}$.

Remark 2: By replacing $\xi_{1}$ by $-\xi_{1}$ in the parametric equations (55), $\Sigma_{m}$ and $\Sigma_{q}$ are changed in $-\Sigma_{m}$ and $-\Sigma_{q}$ when $\tau_{r}=0$. This suggests that the macroscopic yield surface, in the plane $\left(\Sigma_{m}, \Sigma_{q}\right)$, is symmetric with respect to its center $\left(C_{m}, C_{q}\right)$. The representation of this yield surface can then be restricted to the half plane $\left(\Sigma_{m}>0, \Sigma_{q}\right)$ in the absence of residual stresses.

Remark 3: By replacing $\xi_{2}$ by $-\xi_{2}$ in the parametric equations (55), $\Sigma_{m}$ is changed in $-\Sigma_{m}$ while $\Sigma_{s}$ remains unchanged. It follows that the yield locus is symmetric with respect to axis $\Sigma_{s}=0$ in the plane $\left(\Sigma_{m}, \Sigma_{s}\right)$. Noting also that $\Sigma_{s}$ is positive by definition, the yield locus is represented in the quart plane $\left(\Sigma_{m}>0, \Sigma_{s}>0\right)$. This remark also holds for the component $\Sigma_{t}$ at the place of $\Sigma_{s}$.

Remark 4: In the particular case of spherical voids, expressions of $\mathcal{G}_{1}, \mathcal{G}_{2}, \mathcal{G}_{3}$, $\mathcal{G}_{4}$ are given in appendix $\mathrm{C}$. This leads to a parametric equation for the mean stress $\Sigma_{m}$ and equivalent stress $\Sigma_{e q}$ :

$$
\left\{\begin{array}{l}
\Sigma_{m}=\frac{2 \sigma_{0}}{3}\left[\operatorname{arcsinh}\left(\frac{2 u}{f \xi}\right)\right]_{f}^{1}+\frac{2 \tau_{r}}{a_{1}}+\frac{\tau_{0}}{a_{1}} \frac{4}{\sqrt{4+\frac{3 f^{2}}{5} \xi^{2}}} \\
\Sigma_{e q}=-\sigma_{0}\left[\frac{\sqrt{4 u^{2}+f^{2} \xi^{2}}}{u \xi}\right]_{f}^{1}+\frac{\tau_{0}}{a_{1}} \frac{9 \xi f^{2}}{5 \sqrt{4+\frac{3 f^{2}}{5} \xi^{2}}}
\end{array}\right.
$$

where $\xi=D_{e q} / D_{m}$ and $a_{1}$ is the radius of the nanovoid.

By incorporating the effects of interfacial residual stress, (57) extends the results established by Dormieux and Kondo [18] who investigated the case of spherical voids.

\subsection{Closed form expression of the yield function in some particular cases}

- Case 1: $\tau_{0}=0$ 
In this case, the term related to the interfacial plastic strain dissipation, $\Pi_{3}(\boldsymbol{D})$, vanishes in the expression of $\Pi(\boldsymbol{D})$. The macroscopic criterion takes then the form (see appendix $\mathrm{D}$ for more details):

$$
\begin{aligned}
& \frac{\left(\Sigma_{q}-C_{q}-\eta T\right)^{2}}{(1-\zeta) \sigma_{0}^{2}}+\frac{\Sigma_{s}^{2}}{\sigma_{0}^{2}}+\frac{\Sigma_{t}^{2}}{\sigma_{0}^{2}} \\
&+2(1+g)(f+g) \cosh \left(\frac{\kappa T}{\sigma_{0}}\right)-(1+g)^{2}-(f+g)^{2}=0
\end{aligned}
$$

with $T=\Sigma_{m}-C_{m}-\frac{1}{3}\left(1-3 \alpha_{2}\right)\left(\Sigma_{q}-C_{q}\right)$. It is recalled that coefficients $\eta, \zeta$, $\kappa$ and $g$ are given in appendix A.

For the particular case of spherical voids (for which $a_{1}=b_{1}, \zeta=0, \eta=0$, $\kappa=3 / 2$ and $\alpha_{2}=1 / 3$ ), note that the above expression coincides with that given by [18] when the pore space is filled by a fluid and capillary effects develop in the solid/fluid interface (here $\tau_{r}=\gamma^{s f}$, the quantity $\gamma^{s f}$ denoting the surface tension):

$$
\frac{\Sigma_{e q}^{2}}{\sigma_{0}^{2}}+2 f \cosh \left(3 \frac{\Sigma_{m}-2 \tau_{r} / a_{1}}{2 \sigma_{0}}\right)-1-f^{2}=0
$$

Note that (59) reduces to the well-known result of Gurson [35] when $\tau_{r}=0$ or for large values of cavity radius $a_{1}$. In the same vein, when $\tau_{r}=0$ and cavities are spheroidal, (58) reduces to the one established in [49] for a von Mises Matrix (obtained as a particular case of a plastically anisotropic matrix) ${ }^{1}$.

- Case 2: $\frac{\partial \Pi_{1}(\boldsymbol{D})}{\partial(\boldsymbol{D})}<<\frac{\partial \Pi_{2}(\boldsymbol{D})}{\partial(\boldsymbol{D})}$ and $\frac{\partial \Pi_{1}(\boldsymbol{D})}{\partial(\boldsymbol{D})}<<\frac{\partial \Pi_{3}(\boldsymbol{D})}{\partial(\boldsymbol{D})}$

Introducing (37) and (49) into relation (10) yields:

$$
\boldsymbol{\Sigma}=\frac{S_{1}}{V_{1}}\left[\tau_{r} \boldsymbol{N}+\frac{2 \tau_{0}}{3} \frac{\mathbb{S}: \boldsymbol{D}}{\sqrt{\frac{2}{3} \boldsymbol{D}: \mathbb{S}: \boldsymbol{D}}}\right]
$$

with $\boldsymbol{N}$ given by (39) and $\mathbb{S}$ is provided in appendix B.

(60) leads to the following macroscopic elliptic criterion:

$$
\frac{3}{2}\left[\boldsymbol{\Sigma}-\frac{\tau_{r} S_{1}}{V_{1}} \boldsymbol{N}\right]: \mathbb{S}^{-1}:\left[\boldsymbol{\Sigma}-\frac{\tau_{r} S_{1}}{V_{1}} \boldsymbol{N}\right]=\left(\frac{\tau_{0} S_{1}}{V_{1}}\right)^{2}
$$

which is anisotropic due to the presence of directional tensors $N$ and $\mathbb{S}$.

In the case of a hollow sphere, one has $S_{1}=4 \pi a_{1}^{2}, V_{1}=4 \pi a_{1}^{3} / 3, \boldsymbol{N}=2 / 3 \boldsymbol{I}$

$\overline{1}$ Note that the latter has the same form as the GLD-model [31,32] but with other definitions of the coefficients $\eta, \zeta, \kappa, g$. 
and $\mathbb{S}=2 \mathbb{J}+\frac{3 f^{2}}{5} \mathbb{K}$ in which $\mathbb{J}=\frac{1}{3} \boldsymbol{I} \otimes \boldsymbol{I}, \mathbb{K}=\mathbb{I}-\mathbb{J}, \mathbb{I}$ being the fourth order symmetric identity. The macroscopic criterion then reduces to:

$$
\frac{5}{27 f^{2}} \Sigma_{e q}^{2}+\frac{1}{4}\left(\Sigma_{m}-\frac{2 \tau_{r}}{a_{1}}\right)^{2}=\left(\frac{\tau_{0}}{a_{1}}\right)^{2}
$$

- Case 3: $D_{m}=D_{q}=D_{t}=0$

The hollow spheroid is subjected to the following macroscopic shear strain rate loading: $\boldsymbol{D}=D_{12} \underline{e}_{1} \stackrel{s}{\otimes} \underline{e}_{2}$ or $\boldsymbol{D}=D_{11}\left(\underline{e}_{1} \otimes \underline{e}_{1}-\underline{e}_{2} \otimes \underline{e}_{2}\right)$. In that cases, $D_{s}=2\left|D_{12}\right| / \sqrt{3}$ or $D_{s}=2\left|D_{11}\right| / \sqrt{3}$, respectively. The macroscopic criterion corresponding to this shear loading reads:

$$
\Sigma_{s}=(1-f) \sigma_{0}+\frac{\tau_{0} S_{1}}{V_{1}} f \sqrt{\gamma+\frac{\mu}{2}}
$$

It is noted that this result provides an additional interfacial term, $\frac{\tau_{0} S_{1}}{V_{1}} f \sqrt{\gamma+\frac{\mu}{2}}$, comparatively to the GLD-model [31,32] or result by [49]. Note also that the interfacial residual stress $\tau_{r}$ does not affect the above shear strength $\Sigma_{s}$.

- Case 4: $D_{m}=D_{q}=D_{s}=0$

This is similar to the above one and corresponds to the following macroscopic shear strain rate loading, $\boldsymbol{D}=D_{13} \underline{e}_{1} \stackrel{s}{\otimes} \underline{e}_{3}$ or $\boldsymbol{D}=D_{23} \underline{e}_{2} \stackrel{s}{\otimes} \underline{e}_{3}$, for which $D_{t}=2\left|D_{13}\right| / \sqrt{3}$ and $D_{t}=2\left|D_{23}\right| / \sqrt{3}$ respectively. The macroscopic shear strength corresponding to this loading is then given by:

$$
\Sigma_{t}=(1-f) \sigma_{0}+\frac{\tau_{0} S_{1}}{V_{1}} f \sqrt{\frac{5}{2}(1-\gamma)-2 \mu}
$$

\section{Illustrations and validations with numerical calculations}

In this section, we propose to illustrate the salient features of the new macroscopic criterion of a ductile material containing spheroidal nanocavities. In particular, the combined effects of voids size and voids shape will be analyzed. These illustrations are considered for an axisymmetric loading represented by the values of the stress components $\Sigma_{q}=\Sigma_{33}-\Sigma_{11}$ and $\Sigma_{m}=\left(2 \Sigma_{11}+\Sigma_{33}\right) / 3$. We assume that $\Sigma_{s}=\Sigma_{t}=0$, which consists to impose $D_{s}=D_{t}=0$ in the expressions of the macroscopic dissipation and then to put $\xi_{2}=\xi_{3}=0$ in the parametric equations (55). The macroscopic criterion is then described by two parametric equations evolving the macroscopic stress invariants $\Sigma_{m}$ and 
$\Sigma_{t}$ and the parameter $\xi_{1}$.

We will also check the accuracy of the approximate criterion by comparing its predictions to numerical solutions. Indeed, equations (55) have been derived from (53) in which a number of approximations has been used in order to obtain a closed-form expression of the macroscopic dissipation $\Pi(\boldsymbol{D})$. The accuracy of the approximate criterion needs then to be assessed through comparisons with the exact two-field criterion computed numerically. We will first deal with a spherical and then a cylindrical void; the cases of an arbitrary prolate and oblate cavity is thereafter investigated. Finally, we will focus on the case of a penny-shaped crack for which particular attention is needed when computing the limit of $\Pi_{2}(\boldsymbol{D})$ and $\Pi_{3}(\boldsymbol{D})$ when the aspect ratio tends to zero.

\subsection{Case of spherical nano-voids}

\subsubsection{Assessment from atomistic simulations}

In order to investigate the role of the interfacial stress on the macroscopic yielding of plastic materials containing nanovoids we propose a qualitative evaluation of the the interfacial parameters $\tau_{0}$ and $\tau_{r}$. To this end, we refer to the recent study of $\mathrm{Mi}$ et al. [47] who quantify the effect of void radius on the yield strength of a porous cubic single crystal aluminium by making use of atomistic simulations (see also Traiviratana et al. [63] who provide similar results). In this work, they consider a unit cell containing a spherical cavity submitted to a uniaxial tension $E_{33}$, other components of the macroscopic strain remaining equal to zero. Four size for the cavity radius has been considered while the volume fraction of cavity is kept constant and equal to $f=0.0042$. The numerical data in [47] have been reported on figure 6 (the circles) and show the values of the yield strength on the macroscopic yield stress for $\Sigma_{11}$ as function of the radius of the cavity. It must be noted that these results show a strong effect of the cavity radius on the macroscopic yield strength.

We now propose to compare the results obtained by atomistic simulations with the macroscopic model defined by the parametric equations (57). Under uniaxial tension, the strain rate tensor and the stress tensor are on the form: 


$$
\boldsymbol{D}=\left(\begin{array}{ccc}
0 & 0 & 0 \\
0 & 0 & 0 \\
0 & 0 & D_{33}
\end{array}\right), \quad \boldsymbol{\Sigma}=\left(\begin{array}{ccc}
\Sigma_{11} & 0 & 0 \\
0 & \Sigma_{11} & 0 \\
0 & 0 & \Sigma_{33}
\end{array}\right)
$$

For such loading case, $D_{m}=D_{33} / 3$ while $D_{e q}=2 D_{33} / 3$. Parameter $\xi$, which appears in equations (57), takes then the value $\xi=2$. In other hand, the yield stress $\Sigma_{33}$ is given by $\Sigma_{33}=\Sigma_{m}+2 \Sigma_{e q} / 3$ where $\Sigma_{m}$ and $\Sigma_{e q}$ are given by (57). It must be noted that the dependence of $\Sigma_{33}$ with the void radius is on the form $\Sigma_{33}=P+Q / a_{1}$ for which the two constants $P$ and $Q$ are given by:

$$
\left\{\begin{array}{l}
P=\frac{2 \sigma_{0}}{3}\left[\operatorname{arcsinh}\left(\frac{u}{f}\right)-\frac{\sqrt{u^{2}+f^{2}}}{u}\right]_{f}^{1} \\
Q=\frac{2 \tau_{r}}{a_{1}}+\frac{2 \tau_{0}}{a_{1}} \sqrt{1+\frac{3 f^{2}}{5}}
\end{array}\right.
$$

and which are evaluated with the numerical data provided in the paper of $\mathrm{Mi}$ et al. [47]. This computation leads to: $P=2.266 G P a$ and $Q=1.089 G P a$. The volume fraction is known and equal to $f=0.0042$, the value of $\sigma_{0}$ is deduced from the first relation in (66), it gives $\sigma_{0} \simeq 0.59 \mathrm{GPa}$. In (66), the value of $Q$ depends on two unknown material parameters, $\tau_{0}$ and $\tau_{r}$. In order to obtain a qualitative evaluation of $\tau_{0}$ and $\tau_{r}$, we first consider the particular case $\tau_{r}=0$ and then $\tau_{0}=0$. The values of the porosity being very small, it is possible, for this qualitative evaluation of the surface parameters, to neglect the term $3 f^{2} / 4$ under the square root in the expression of $Q$. This coefficient is then approximated by:

$$
Q=\frac{2 \tau_{r}}{a_{1}}+\frac{2 \tau_{0}}{a_{1}}
$$

Note that in the above expression the effect of $\tau_{0}$ and $\tau_{r}$ on coefficient $Q$ is the same. If $\tau_{r}=0$, one has $\tau_{0}=0.545 \mathrm{~N} / \mathrm{m}$. The second case, $\tau_{0}=0$, leads to the same value for $\tau_{r}$. Note that the computation of both $\tau_{0}$ and $\tau_{r}$ could be done by performing the atomistic simulation in the case of uniaxial compression, indeed, it will be recalled that the term containing $\tau_{r}$ introduces an asymmetry between tension and compression.

In figure 6, the numerical data obtained from atomistic simulations (the circles) are compared to the parametric criterion (57) with the calibrated material parameters $\tau_{0}$ and $\tau_{r}$ (the full line). Just as information, we also represent the prediction of the Gurson model, which do not account for the size effect, and which corresponds to $\Sigma_{33}=P$. It must be noted that the model can reproduce 
the dependence of the yield strength with the cavity radius in the particular case of spherical shape.

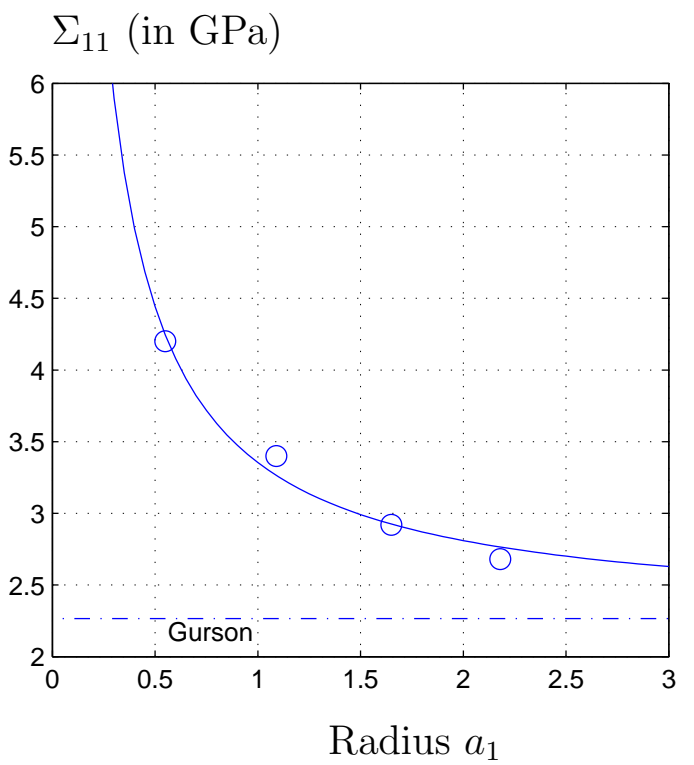

Fig. 6. Yield strength for $\Sigma_{11}$ as function of the radius of the cavity. Comparison between the numerical date obtained form atomistic simulation by Mi et al. [47] (circles), the macroscopic model $\Sigma_{11}=P+Q / a_{1}$ with optimized value values for $P$ and $Q$ (the full line) and the Gurson prediction (dashed line).

\subsubsection{Representation of the macroscopic criterion}

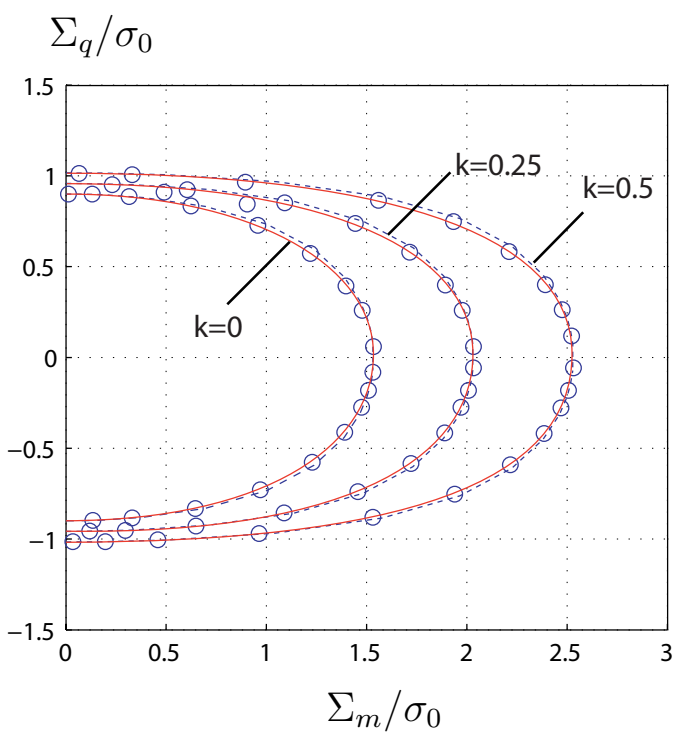

Fig. 7. Yield loci for spherical nanovoids. Comparison between the approximate criterion (dashed line) and the exact, numerical two-field criteria (full line with circles) for a porosity $f=0.1$ and for various values of $k=\tau_{0} /\left(a_{1} \sigma_{0}\right)$. 
Figure 7 illustrates the particular case of spherical nano-cavities for which the approximate yield surface is given by the parametric equation (55). Two values of the non dimensional parameter $k=\tau_{0} /\left(a_{1} \sigma_{0}\right)$, namely $k=0.25$ and $k=0.5$, as well as a fixed porosity $f=0.1$ and $\tau_{r}=0$ are considered. By using the values $\tau_{0}=0.54 \mathrm{~N} / \mathrm{m}$ and $\sigma_{0}=0.59 \mathrm{GPa}$, the cases $k=0.25$ and $k=0.5$ correspond to a cavity of radius $1.83 \mathrm{~nm}$ and $3.66 \mathrm{~nm}$ respectively. The results show an important effect of the parameter $k$ on the macroscopic yield locus: the yield strength domain increases when the voids size decreases. In this figure, a very good agreement is also observed between the approximate criterion and the results obtained by numerically evaluating the macroscopic dissipation (15).

\subsection{Case of cylindrical nano-cavities}

The case of a hollow cylinder corresponds to the limits $a_{1} \rightarrow \infty$ and $a_{2} \rightarrow \infty$, that is $e_{1} \rightarrow 1$ and $e_{2} \rightarrow 1$. On figure 8 is represented the macroscopic yield function in the plane $\left(\Sigma_{m}, \Sigma_{q}\right)$ for a porosity $f=0.1$ and for various values of the non dimensional parameter $k=\tau_{0} /\left(b_{1} \sigma_{0}\right)$; the residual stress $\tau_{r}=0$ for this illustration. These results show an important effect of the cavity size on the macroscopic yield locus, similar to that observed for spherical voids.

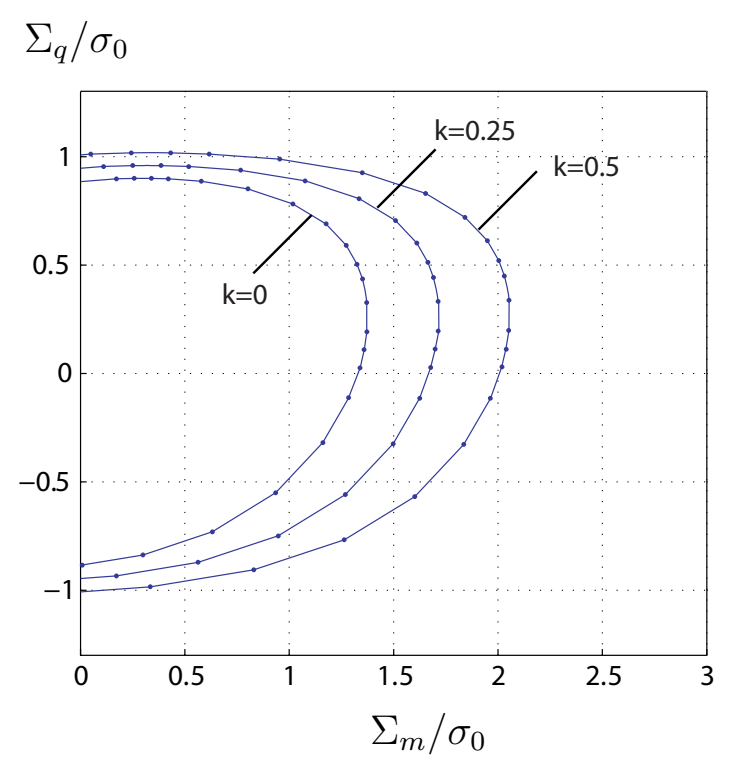

Fig. 8. Yield loci for the ductile material with a cylindrical nanovoid for a porosity $f=0.1$ and for various values of $k=\tau_{0} /\left(b_{1} \sigma_{0}\right)$. 


$$
\Sigma_{q} / \sigma_{0}
$$



$\Sigma_{q} / \sigma_{0}$



Fig. 9. Yield loci for a prolate cavity with an aspect ratio $a_{1} / b_{1}=2$ (at the left) $a_{1} / b_{1}=5$ (at the right) and a porosity $f=0.1$. Comparison between the approximate (full line with points) and the exact, numerical, two-field criteria (circles) for various values of $k=\tau_{0} /\left(b_{1} \sigma_{0}\right)$.

\subsection{Ductile materials with spheroidal nanovoids}

On Figure 9, is represented the macroscopic yield surface for the case of a prolate void. On the left, this yield surface is plotted for an aspect ratio $a_{1} / b_{1}=2$ and a porosity $f=0.1$, while, at the right, the figure corresponds to $a_{1} / b_{1}=5$ and the same porosity. $k$ is the non dimensional parameter defined as $k=\tau_{0} /\left(b_{1} \sigma_{0}\right)$. To check the accuracy of the approximate criterion (full line with the points), we compare the results with numerical data of the exact two-field criterion (circles): a very good agreement is observed. Figure 10 displays similar results for the case of an oblate cavity. At the right, the aspect ratio is $a_{1} / b_{1}=1 / 2$ while at the left, an aspect ratio of $a_{1} / b_{1}=1 / 5$ is considered. A very good agreement between the "approximate" and the "exact" two-field criterion is observed for low values of the parameter $k=\tau_{0} /\left(b_{1} \sigma_{0}\right)$. When increasing $k$, some slight differences are noted in the region of positive values of $\Sigma_{q}$ and high values of the mean stress $\Sigma_{m}$. Beyond that differences, it can be also noted that the approximate criterion seems to preserve the upper bound character of the limit analysis-based approach, since the predictions of the approximate two-field criterion are larger than the numerical ones.

We now represent on figure 11 the yield strength of the nanoporous medium as function of the parameter $k=\tau_{0} /\left(b_{1} \sigma_{0}\right)$. At the right, we represent the deviatoric yield strength, $\Sigma_{q}$ (other components of the macroscopic stress are null), obtained by putting $\xi_{2}=\xi_{3}=0$ and $\xi_{1}=+\infty$ in the parametric equations (55). A porosity $f=0.1$ is considered and we compare the predictions 

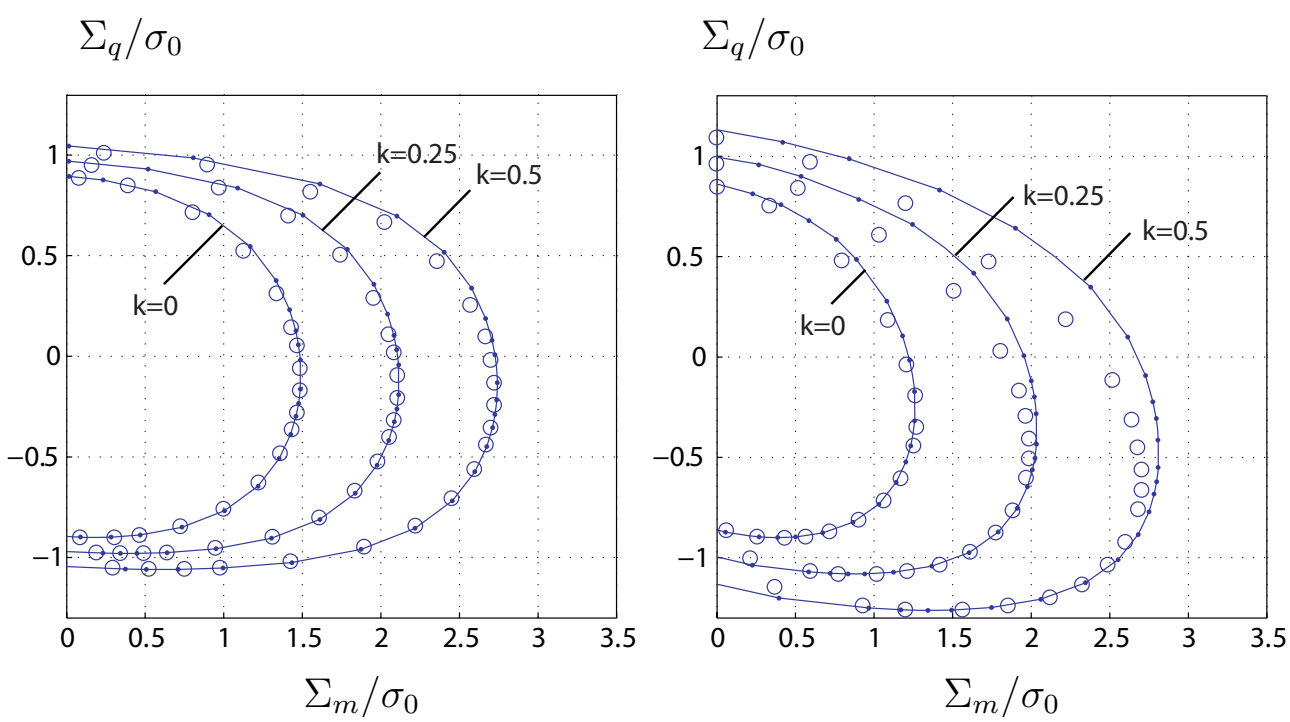

Fig. 10. Yield loci for a oblate cavity with an aspect ratio $a_{1} / b_{1}=1 / 2$ (at the left) $a_{1} / b_{1}=1 / 5$ (at the right) and a porosity $f=0.1$. Comparison between the approximate (full line with points) and the exact, numerical, two-field criteria (circles), for various values of $k=\tau_{0} /\left(b_{1} \sigma_{0}\right)$.

for a prolate cavity (with the aspect ratio $a_{1} / b_{1}=5$ ) with those obtained for an oblate one (with the aspect ratio $a_{1} / b_{1}=1 / 5$ ). Also, in each case, the approximate value for the plastic limit is compared to the numerical exact one. The symbols used on figure 11 are the same as that of figures 9 and 10: the full line with the points corresponds to the approximate solution, whereas the circles are associated to the numerical two-field estimate. On the left, we present similar results for $\Sigma_{m}$ (other components of the macroscopic stress are null), obtained by putting $\xi_{1}=\xi_{2}=\xi_{3}=0$ in the parametric equations (55). As expected, the yield strength increase when decreasing the voids size. It is worth noticing that the voids size effects seem to be more important in the case of an oblate cavity than for a prolate one. The reason is that for the same void porosity, the area of the void surface is greater for an oblate cavity than for a prolate one.

For completeness, we now propose to evaluate the effect of the interfacial residual stresses on the macroscopic yield surface of the nanoporous material. To this end, we come back to the case $\tau_{0}=0$ which leads to the closed-form expression (58). On figure 12, on the left, are represented the yield surfaces for a prolate cavity with an aspect ratio $a_{1} / b_{1}=1 / 2$, a porosity $f=0.1$ and two values for the non dimensional parameter $k_{r}=\tau_{r} /\left(b_{1} \sigma_{0}\right)$. On the right, are represented similar results for the case of an oblate cavity having an aspect ratio $a_{1} / b_{1}=1 / 5$. A significant influence of the residual stress on the yield strength is observed. More precisely, the residual stresses induce a translation of the center of the macroscopic yield surface along both axis related to the macroscopic stress invariants $\Sigma_{m}$ and $\Sigma_{q}$. This imply an asymmetry of the macroscopic yield strength between tension and compression. Again, it is 

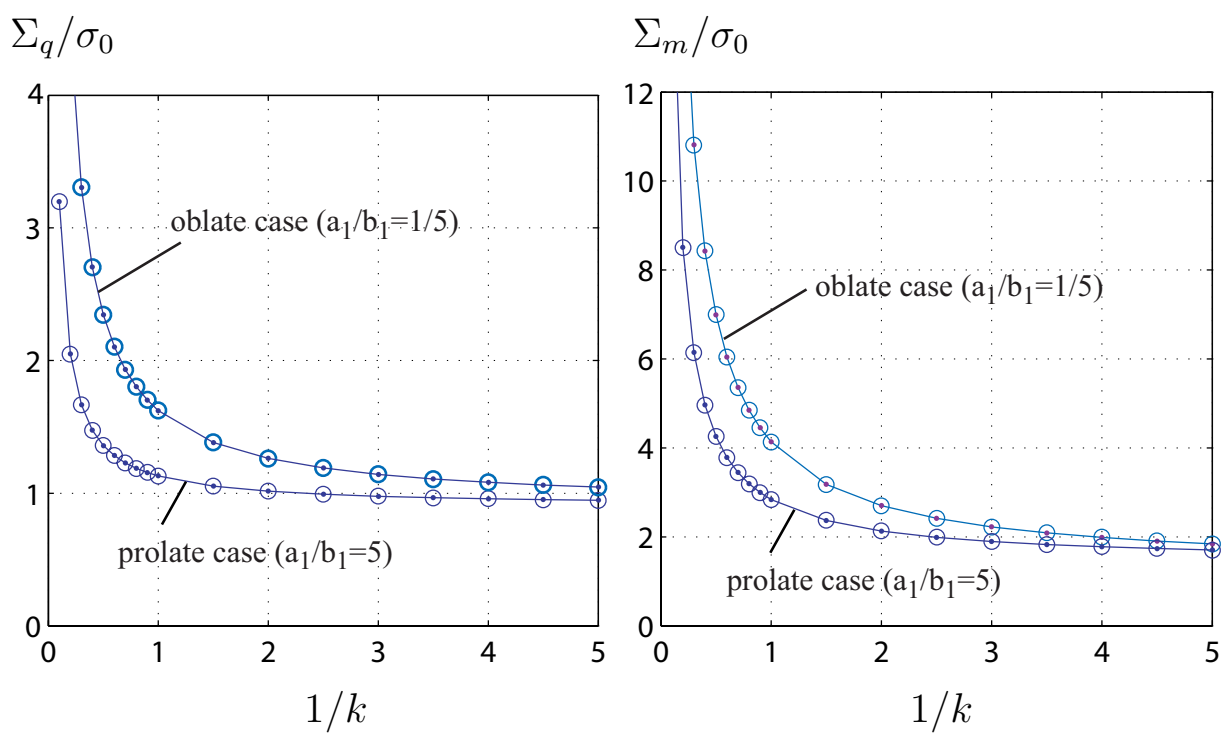

Fig. 11. On the left: variations of the shear yield strength for $\Sigma_{q}$ as function of $1 / k=b_{1} \sigma_{0} / \tau_{0}$. At the right: variations of the yield strength for $\Sigma_{m}$ as function of $1 / k=b_{1} \sigma_{0} / \tau_{0}$. The considered porosity is $f=0.1$ and the aspect ratios are $a_{1} / b_{1}=5$ (prolate case) and $b_{1} / a_{1}=5$ (oblate case). The full line represents the approximate solution, the discrete points (circles) the numerical exact two-field solution.
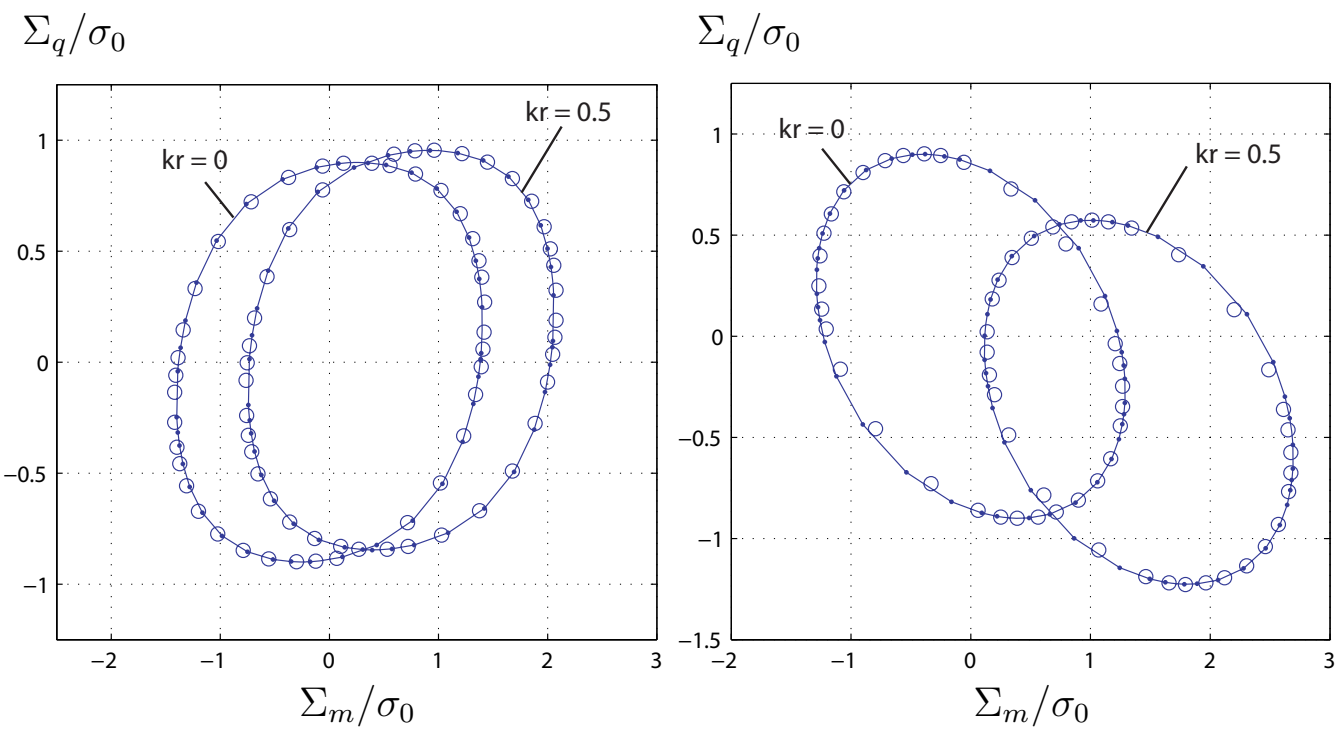

Fig. 12. Yield loci for a prolate cavity with an aspect ratio $a_{1} / b_{1}=5$ (on the left) for an oblate cavity with $a_{1} / b_{1}=1 / 5$ (on the right), a porosity $f=0.1$ and for two values of the parameter $k_{r}=\tau_{r} /\left(b_{1} \sigma_{0}\right)$.

observed that the interfacial stress effects are more pronounced for an oblate cavity than for a prolate one (for the same reasons mentioned above). 


\subsection{The particular case of nano penny-shaped cracks}

We consider now the case of a nanosized penny-shaped crack, corresponding to an oblate spheroidal nanocavity with an aspect ratio $\epsilon=a_{1} / b_{1} \rightarrow 0$ or equivalently with an eccentricity $e_{1} \rightarrow 1$. Since the volume $V_{1}$ of the cavity tends to zero, the ratio $\frac{\tau_{0} S_{1}}{V_{1}}$ which appears in expression of $\Pi_{2}(\boldsymbol{D})$ and $\Pi_{3}(\boldsymbol{D})$, respectively given by (40) and (51), diverge. In the other hand, coefficients $h_{n}$ for $n=1 . .5$ which enters under the square root in (51) (and given by (52)) as well as the coefficients $g_{n}$ which appears in (40) (and given by (41)) tend to zero when the limit $e_{1} \rightarrow 1$ is considered. However, it can be shown that $\Pi_{2}(\boldsymbol{D})$ and $\Pi_{3}(\boldsymbol{D})$ have a finite limit when $e_{1} \rightarrow 1$. Indeed, in the case of a penny-shaped crack, coefficients $h_{n}$ are proportional to $\epsilon^{2}$ and $g_{n}$ are proportional to $\epsilon$ while the ratio $\frac{\tau_{0} S_{1}}{V_{1}}$ is proportional to $\frac{1}{\epsilon}$. By computing carefully the expressions of the dissipation $\Pi_{2}(\boldsymbol{D})$ and $\Pi_{3}(\boldsymbol{D})$, we get:

$$
\begin{aligned}
\Pi_{2}(\boldsymbol{D}) & =\frac{3 \tau_{r}}{2 b_{1}}\left(\tilde{g}_{1} D_{m}+\tilde{g}_{2} D_{q}\right) \\
\Pi_{3}(\boldsymbol{D}) & =\frac{3 \tau_{0}}{2 b_{1}} \sqrt{\tilde{h}_{1} D_{m}^{2}+\tilde{h}_{2} D_{q}^{2}+2 \tilde{h}_{3} D_{m} D_{q}+\tilde{h}_{4} D_{s}^{2}}
\end{aligned}
$$

where coefficients $\tilde{g}_{n}$ for $n=1,2$ are obtained by taking the limit of $g_{n} / \epsilon$ for $\epsilon \rightarrow 0$, while $\tilde{h}_{n}$ for $n=1,2,3,4$ are obtained as the limit of $h_{n} / \epsilon^{2}$ for $\epsilon \rightarrow 0$. Note that term involving $D_{t}$ does not appear in $\Pi_{3}(\boldsymbol{D})(68)$; this is due to the fact that the limit $h_{5} / \epsilon^{2}$ for $\epsilon \rightarrow 0$ is zero. The final expressions for $\tilde{g}_{n}$ and $\tilde{h}_{n}$ are:

$$
\left\{\begin{array}{l}
\tilde{g}_{1}=\frac{3 \pi}{2}-\left(1-3 \alpha_{2}\right) d \\
\tilde{g}_{2}=d \\
\tilde{h}_{1}=24+\frac{9 \pi^{2}}{4}-3 \pi\left(1-3 \alpha_{2}\right) d+\left(1-3 \alpha_{2}\right)^{2} d^{2} \\
\tilde{h}_{2}=d^{2} \\
\tilde{h}_{3}=\frac{1}{2}\left(3 \pi-2 d\left(1-3 \alpha_{2}\right)\right) d \\
\tilde{h}_{4}=d^{2}
\end{array}\right.
$$

in which $d$ is the crack density parameter, $d=b_{1}^{3} /\left(a_{2} b_{2}^{2}\right)$, introduced first by Bristow [8] and later considered by Budiansky and O'Connell [9] when studying elastic properties of cracked media. In figure 13, we represent the macroscopic criterion for a penny-shaped crack having a density parameter $d=0.2$; various values of the non dimensional parameter $k=\tau_{0} /\left(b_{1} \sigma_{0}\right)$ and 


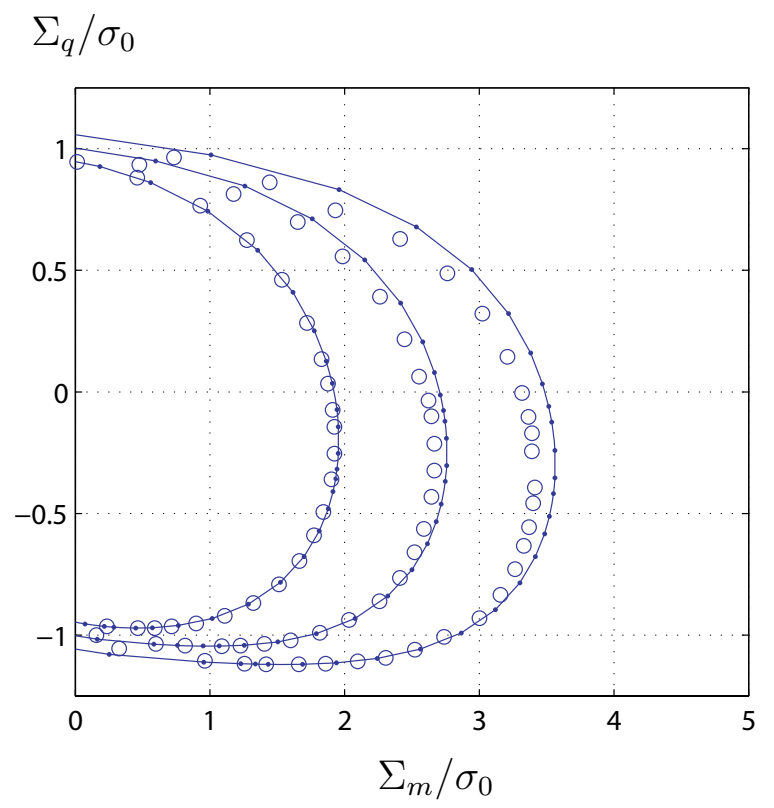

Fig. 13. Yield loci for a nano penny-shaped crack. Comparison between the approximate (full line with points) and the exact numerical two-field criteria (circles) for a crack density parameter $d=0.2$ and for various values of $k=\tau_{0} /\left(b_{1} \sigma_{0}\right)$.

$\tau_{r}=0$ are considered. Again, it can be observed that the approximate criterion (full line with points) compare well with the exact two-field criterion (circles).

\section{Conclusion}

The present paper has been devoted to the study of anisotropic ductile nanoporous materials. Particular emphasis has been put on interfacial stress and its influence on the overall yield criterion. The combined effects of void shape and size are investigated by considering prolate and oblate cavities with surface stresses. The theoretical development has been carried out by making use of limit analysis of a spheroidal unit cell made up of a von Mises solid matrix containing a confocal spheroidal cavity. To this end, the trial velocity field, introduced by Gologanu et al. [31,32], has been considered in order to derive the macroscopic yield criterion.

An original aspect of the analysis lies in the incorporation of a stress interface (between void and matrix) which accounts for a jump of the traction vector across the surface of the void. To this end, we took advantage of the plastic version of the Gurtin model, recently proposed by Monchiet and Bonnet [50], in which the jump of the traction vector at the void-matrix interface results from two contributions: surface residual stresses and plastic deformation at the void surface. In addition to the classical volumetric dissipation in the matrix, two different contributions to the macroscopic dissipation have been 
identified; they correspond to interfacial residual stresses and plastic strain rates, respectively. Closed-form expressions of these interfacial contributions have been derived. This allowed to determine approximate parametric equations of the macroscopic yield function which, interestingly, points out the crucial role of the ratio between the void surface and the cell volume. These results extend those recently established by [18] which deal with ductile materials containing spherical nanovoids and in which voids size effects have been also demonstrated. In addition to the above results, numerical calculations, based on the considered two-field trial velocity have been performed in order to derive an upper bound for the macroscopic yield criterion. Illustrations has been provided for various shape of the cavity and various values of the material parameters which enter into the stress interface model. It has been shown that the stress interface has a significant effect on the macroscopic yield surface of the ductile material containing spheroidal nanocavities. In particular, the surface plastic deformation induces, at the macroscopic level, an increase of the yield strength. Concerning the interfacial residual stress, it leads to a change in position of the macroscopic yield surface, resulting in an asymmetry between tension and compression. Note that a good agreement has been noted between the predictions of the approximate parametric equations and that of the numerical upper bound. Finally, it has been shown that the surface effects on the macroscopic yield strength are more pronounced for an oblate cavity than for a prolate one.

\section{References}

[1] R. Agrawal, B. Peng, E.E. Gdoutos, H.D. Espinosa. Elasticity size effects in $\mathrm{ZnO}$ nanowires - a combined experimental-computational approach. Nano Letter. 8(11), 3668-3674, 2008.

[2] M.F. Ashby. The deformation of plastically non-homogeneous alloys. Philosophical Magazine. 21, 399-424, 1970.

[3] Y. Benveniste, G.W. Milton. New exact results for the e'ective electric, elastic, piezoelectric and other properties of composite ellipsoid assemblages. Journal of the Mechanics and Physics of Solids, 51, 1773-1813, 2003.

[4] Y. Benveniste. A general interface model for a three-dimensional curved thin anisotropic interphase between two anisotropic media. Journal of the Mechanics and Physics of Solids, 54, 708-734, 2006.

[5] A. Benzerga and J.B. Leblond. Ductile Fracture by Void Growth to Coalescence. Advance in Applied Mechanics, 44 : 169-305, 2010.

[6] S. Brisard, L. Dormieux, D. Kondo. Hashin-Shtrikman bounds on the bulk modulus of a nanocomposite with spherical inclusions and interface effects. Computational Materials Science, 48, 589-596, 2010. 
[7] S. Brisard, L. Dormieux, D. Kondo. Hashin-Shtrikman bounds on the bulk modulus of a nanocomposite with spherical inclusions and interface effects. Computational Materials Science, 50, 403-410, 2010.

[8] J.R. Bristow. Microcracks and the static and dynamic elastic constants of annealed heavily cold-worked metals. British Journal of Applied Physics, 11, 81-85, 1960.

[9] B. Budiansky, R.J. O'connell. Elastic moduli of a cracked solid. International Journal of Solids Structures, 12(2), 81-97, 1976.

[10] M. Cadek, J.N. Coleman, K.P. Ryan, V. Nicolosi, G. Bister, A. Fonseca, J.B. Nagy, K. Szostak, F. Beguin, W.J. Blau. Reinforcement of polymers with carbon nanotubes: the role of nanotube surface area. Nano Letters. 4, 353-356, 2004.

[11] W.-H. Chen, H.-C. Cheng, Y.-C. Hsu, R.-H. Uang, J.-S. Hsu. Mechanical material characterization of Co nanowires and their nanocomposites, Composites Science Technology. 68, 2288-3395, 2008.

[12] A.H. Cottrell. The Mechanical Properties of Matter. J. Wiley, New York, 1964.

[13] S. Cuenot, C. Frétigny, S. Demoustier-Champagne, B. Nysten. Surface tension effect on the mechanical properties of nanomaterials measured by atomic force microscopy. Physical Review B. 69, 165410, 2004.

[14] J.K. Diao, K. Gall, M.L. Dunn. Atomistic simulation of the structure and elastic properties of gold nanowires. Journal of the Mechanics Physics and Solids, 52, 1935-1962, 2004.

[15] J. Diao, K. Gall and M.L. Dunn. Yield Strength Asymmetry in Metal Nanowires. Nano Letters, 4(10), 1863-1867, 2004.

[16] J.K. Diao, K. Gall, M.L. Dunn, J.A. Zimmerman. Atomistic simulations of the yielding of gold nanowires. Acta Materialia, 54, 643-653, 2006.

[17] R. Dingreville, J. Qiu, M. Cherkaoui. Surface free energy and its effect on the elastic behavior of nano-sized particles, wires and films. Journal of the Mechanics Physics and Solids, 53, 1827-1854, 2005.

[18] L. Dormieux, D. Kondo. An extension of Gurson model incorporating stresses effects. International Journal of Engineering Science, 48, 575-581, 2010.

[19] L. Duan, J. Wang, Z.P. Huang, B.L. Karihaloo Size-dependent effective elastic constants of solids containing nano-inhomogeneities with interface stress. Journal of the Mechanics Physics and Solids, 53, 1574-1596, 2005.

[20] H.L. Duan, J. Wang, Z.P. Huang and B.L. Karihaloo. Eshelby formalism for nano-inhomogeneities. Proceedings of the Royal Society A, 461, 3335-3353, 2005. 
[21] H.L. Duan, X. Yi, Z.P. Huang, J. Wang. A unified scheme for prediction of effective moduli of multiphase composites with interface effects. Part I: Theoretical framework. Mechanics of Materials, 39, 81-93, 2007.

[22] H.L. Duan, X. Yi, Z.P. Huang, J. Wang. A unified scheme for prediction of effective moduli of multiphase composites with interface effects. Part II: application and scaling laws. Mechanics of Materials, 39, 94-103, 2007.

[23] N.A. Fleck, G.M. Muller, M.F. Ashby, J.W. Hutchinson. Strain gradient plasticity: theory and experiment. Acta Metall. Mater. 42(2), 475-487, 1994.

[24] N.A. Fleck, J.W. Hutchinson. Strain gradient plasticity. In: Hutchinson, J.W., Wu, T.Y. (Eds.), Advance in Applied Mechanics, 33. Academic Press, New York, 295-361, 1997.

[25] N.A. Fleck, J.W. Hutchinson. A reformulation of strain gradient plasticity. Journal of the Mechanics Physics and Solids, 49, 2245-2271, 2001.

[26] K. Gall, J. Diao, M.L. Dunn. The Strength of Gold Nanowires. Nano Letters, 4(12), 2431-2436, 2004.

[27] H. Gao, Y. Huang, W.D. Nix. Modeling plasticity at the micrometer scale. Naturwissenschaften 86, 507-515, 1999.

[28] H. Gao, Y. Huang. Taylor-based nonlocal theory of plasticity. International Journal of Solids and Structures, 38, 2615-2637, 2001.

[29] M. Gãrãjeu. Contribution à l'étude du comportement non linéaire de milieux poreux avec ou sans renfort. Thèse de doctorat de l'Université de Marseille, 1995.

[30] M. Garajeu, J.C. Michel, P. Suquet. A micromechanical approach of damage in viscoplastic materials by evolution in size, shape and distribution of voids. Computational Methods in Applied Mechanics and Engineering, 183, 223-246, 2000.

[31] M. Gologanu, J.-B. Leblond, J. Devaux. Approximate models for ductile metals containing non-spherical voids - case of axisymmetric prolate ellipsoidal cavities. Journal of the Mechanics Physics and Solids, 41 (11), 1723-1754, 1993.

[32] M. Gologanu, J.B. Leblond, G. Perrin, J. Devaux. Approximate models for ductile metals containing non-spherical voids - case of axisymmetric oblate ellipsoidal cavities. Journal of Engineering Materials and Technology, 116, 290297, 1994.

[33] M. Gologanu, J.B. Leblond, G. Perrin and J. Devaux. Recent extensions of Gurson's model for porous ductile metals. Continuum Micromechanics, ed. P. Suquet, Springer Verlag, 1997.

[34] T. Goudarzi, R. Avazmohammadi, R. Naghdabadi. Surface energy effects on the yield strength of nanoporous materials containing nanoscale cylindrical voids. Mechanics of Materials, 42(9), 852-862, 2010. 
[35] A.L. Gurson. Continuum theory of ductile rupture by void nucleation and growth: Part I. - Yield criterion and flow rules for porous ductile media. Journal of Engineering Materials and Technology, 99, 2-15, 1977.

[36] M.E. Gurtin, A.I. Murdoch. A continuum theory of elastic material surfaces. Archive for Rational Mechanics and Analysis, 57, 291-323, 1975.

[37] M.E. Gurtin, A.I. Murdoch. Surface stress in solids. International Journal of Solids and Structures, 14, 431-440, 1978.

[38] J.C.Hamilton, W.G. Wolfer. Theories of surface elasticity for nanoscale objects, Surface Science, 603(9), 1284-1291, 2009.

[39] M. Huang, Z. Li, C. Wang. Coupling effects of void size and void shape on the growth of prolate ellipsoidal microvoid. Acta Mechanica Sinica, 21, 272-277, 2005 .

[40] M. Huang, Z. Li. Size effects on stress concentration induced by a prolate ellipsoidal particle and void nucleation mechanism. International Journal of Plasticity, 21(8), 1568-1590, 2005.

[41] Y. Huang, X. Bai, Y. Zhang. In situ mechanical properties of individual $\mathrm{ZnO}$ nanowires and the mass measurement of nanoparticles. Journal of Physics and Condensed Matter, 18, 179-184, 2006.

[42] H. Le Quang, Q.-C. He. Size-dependent effective thermoelastic properties of nanocomposites with spherically anisotropic phases. Journal of the Mechanics and Physics of Solids, 55(9), 1899-1931, 2007.

[43] Z. Li, M. Huang. Combined effects of void shape and void size - oblate spheroidal microvoid embedded in infinite non-linear solid. International Journal of Plasticity, 21(3), 625-650, 2005.

[44] Z. Li, P. Steinmann. RVE-based studies on the coupled effects of void size and void shape on yield behavior and void growth at micron scales. International Journal of Plasticity, 22(7), 1195-1216, 2006.

[45] B. Liu, Y. Huang, M. Li, K.C. Hwang, C. Liu. A study of the void size effect based on the Taylor dislocation model. International Journal of Plasticity, 21(11), 2107-2122, 2005.

[46] P.E. Marszalek, W.J. Greenleaf, H. Li, A.F. Oberhauser, J.M. Fernandez. Atomic force microscopy captures quantized plastic deformation in gold nanowires. Proceedings of the National Academy of Sciences of the United States of America, 97, 6282, 2000.

[47] C. Mi, D.A. Buttry, P. Sharma, D.A. Kouris. Atomistic insights into dislocation-based mechanisms of void growth and coalescence. Journal of the Mechanics and Physics of Solids, 59, 1858-1871, 2011.

[48] R.E. Miller, V.B. Shenoy. Size-dependent elastic properties of nanosized structural elements. Nanotechnology, 11, 139-147, 2000. 
[49] V. Monchiet, O. Cazacu, E. Charkaluk, D. Kondo. Approximate criteria for anisotropic metals containing non spherical voids. International Journal of Plasticity, 24, 1158-1189, 2008.

[50] V. Monchiet, G. Bonnet. Interfacial models in viscoplastic composites materials. International Journal of Engineering Science, 48(12), 1762-1768, 2010 .

[51] V. Monchiet, E. Charkaluk, D. Kondo. A Micromechanics-based modification of the Gurson criterion by using Eshelby-like velocity fields. European Journal of Mechanics - A/Solids, 30, 940-949, 2011.

[52] V. Monchiet, E. Charkaluk, D. Kondo. Macroscopic yield criteria for ductile materials containing spheroidal voids: an Eshelby-like velocity fields approach. Submitted.

[53] J.F. Nye. Some geometrical relations in dislocated crystals. Acta Matallurgica, 1, 153-162, 1953.

[54] Z.Y. Ou, G.F. Wang, T.J. Wang. Effect of residual tension on the stress concentration around a nanosized spheroidal cavity, Int. J. Engng. Science. 46(5), 475-485, 2009.

[55] Z.Y. Ou, G.F. Wang, T.J. Wang. Elastic fields around a nanosized spheroidal cavity under arbitrary uniform remote loadings, Eur. J. Mech. A. Solid, 28, 110-120, 2009.

[56] H.S. Park, J.A. Zimmerman. Modeling inelasticity and failure in gold nanowires. Physical Review B, 72(5), 054106, 2005.

[57] H.S. Park, K. Gall, J.A. Zimmerman. Deformation of FCC nanowires by twinning and slip. Journal of the Mechanics Physics and Solids, 54, 1862-1881, 2006 .

[58] Y.Z. Povstenko. Theoretical investigation of phenomena caused by heterogeneous surface tension in solids. Journal of the Mechanics Physics and Solids, 41, 1499-1514, 1993.

[59] A.R. Sethoodeh, H. Attariani, M. Khosrownejad. Nickel nanowires under uniaxial loads: a molecular dynamics simulation study. Computer Material Science, 44, 378-384, 2008.

[60] P. Sharma, S. Ganti Size-dependent Eshelby's tensor for embedded nanoinclusions incorporating surface/interface energies. ASME Journal of Applied Mechanics, 71, 663-671, 2004.

[61] V.B. Shenoy. Atomistic calculations of elastic properties of metallic fcc crystal surfaces, Phys. Rev., B 71:094104-1-11, 2005.

[62] D.J. Steigmann, R.W. Ogden. Plane deformations of elastic solids with intrinsic boundary elasticity. Proceeding of the Royal Society of London A, 453, 853-877, 1997. 
[63] S. Traiviratana, E.M. Bringa, D.J. Benson, M.A. Meyers. Void growth in metals: Atomistic calculations. Acta Materialia, 56, 3874-3886, 2008.

[64] V. Tvergaard, C. Niordson. Nonlocal plasticity effects on interaction of different size voids. International Journal of Plasticity, 20, 107-120, 2004.

[65] L.J. Walpole. Elastic behavior of composite materials : theoretical foundations. Advances in Applied Mechanics, 21, 169-243, 1981.

[66] G. Wang, X. Li. Predicting Young's modulus of nanowires from first-principles calculations on their surface and bulk materials. Journal of Applied Physics, 104, 113517, 2008.

[67] J. Wen, Y. Huang, K.C. Hwang, C. Liu, M. Li. The modified Gurson model accounting for the void size effect. International Journal of Plasticity, $21(2), 381-395,2005$.

[68] E.W. Wong, P.E. Sheehan, C.M. Lieber. Nanobeam mechanics: elasticity, strength, and toughening of nanorods and nanotubes. Science, 277, 1971-1975, 1997.

[69] B. Wu, A. Heidelberg, J.J. Boland. Mechanical properties of ultrahighstrength gold nanowires. Nature Materials, 4, 525-529, 2005.

[70] Z. Yang, Z. Lu, Y.-P. Zhao. Atomistic simulation on size-dependent yield strength and defects evolution of metal nanowires. Computational Materials Science, 46, 142-150, 2009.

[71] J. Yvonnet, A. Mitrushchenkov, G. Chambaud, Q.-C. He. Finite element model of ionic nanowires with size-dependent mechanical properties determined by ab initio calculations. Computer Methods in Applied Mechanics and Engineering, 200, 614-625, 2011.

[72] W. Zhang, T.J. Wang. Effect of surface energy on the yield strength of nanoporous materials. Applied Physics Letters, 90, 063104, 2007.

[73] W. Zhang, T.J. Wang, X. Chen. Effect of surface stress on the asymmetric yield strength of nanowires. Journal of Applied Physics, 103, 123527, 2008.

[74] W. Zhang, T.J. Wang, X. Chen. Effect of surface/interface stress on the plastic deformation of nanoporous materials and nanocomposites. International Journal of Plasticity, 26(7), 957-975, 2010.

\section{A Coefficients in $\Pi_{1}(\boldsymbol{D})$}

In this appendix are provided the coefficients which enter in the closed-form expression of the plastic dissipation $\Pi_{1}(\boldsymbol{D})$ given by (30). Expressions of coefficients $\eta$ and $\zeta$ are 


$$
\eta=\frac{\kappa^{2}(1+g)(f+g)\left(\alpha_{2}-\alpha_{1}\right)}{(1-f)} ; \quad \zeta=\frac{\kappa^{2}(1+g)(f+g)\left(\alpha_{2}-\alpha_{1}\right)^{2}}{(1-f)^{2}}
$$

where $g$ is defined by:

$$
g=\frac{c^{3}}{a_{2} b_{2}^{2}}=\frac{e_{2}^{3}}{\sqrt{1-e_{2}^{2}}}
$$

and coefficient $\kappa$ is defined by:

$$
\begin{aligned}
& \kappa=\frac{1}{\ln (f)} \int_{e_{1}}^{e_{2}}\left[3\left(1+3 \alpha^{2}\right)+\frac{6(1-3 \alpha)}{3-2 e^{2}}\right] \frac{3-2 e^{2}}{e\left(1-e^{2}\right)} d e \quad \text { (prolate) } \\
& \kappa=\frac{1}{\ln \left(\frac{g+f}{1+g}\right)} \int_{e_{1}}^{e_{2}}\left[3\left(1+3 \alpha^{2}\right)+\frac{6(1-3 \alpha)\left(1-e^{2}\right.}{3-e^{2}}\right] \frac{3-e^{2}}{\left.e\left(1-e^{2}\right)\right)} d e
\end{aligned}
$$

$u_{1}$ and $u_{2}$ are given by:

$$
u_{1}=\frac{f}{1+g}, \quad u_{2}=\frac{f}{f+g}
$$

where $g=0$ for the case of a prolate cavity and $g$ is given by (A.2) for the case of an oblate cavity.

\section{B Computation of $\bar{P}, \overline{\mathbb{P}}$ and $\mathbb{S}$}

Performing the integral for $\theta$ over $[0,2 \pi]$ in (34), it comes:

$$
\int_{\theta=0}^{\theta=2 \pi} \boldsymbol{P}(\theta, \varphi)=\pi\left(1+w^{2}\right) \boldsymbol{I}_{t}+\pi\left(1-w^{2}\right) \boldsymbol{I}_{n}
$$

where $w$ is defined by:

$$
w=\frac{b \cos (\varphi)}{L_{\lambda}}
$$

For computing the second integral in (34), according to $\varphi$ over the interval $[0, \pi]$, one can use the following result:

$$
\frac{2 \pi}{S_{1}} \int_{\varphi=0}^{\varphi=\pi} w^{2} b_{1} L_{\lambda_{1}} \sin (\varphi) d \varphi=\gamma
$$


Tensor $\overline{\mathbb{P}}$ is a transversely isotropic (with respect to the $O x_{3}$ axis) and symmetric fourth order tensor. This tensor is then a priori defined by 5 independent coefficients. Moreover, by considering the following equalities:

$$
\begin{aligned}
& \bar{P}_{1111}+\bar{P}_{1122}+\bar{P}_{1133}=\frac{1}{3} \bar{P}_{11}=\frac{1-\gamma}{6} \\
& 2 \bar{P}_{1133}+\bar{P}_{3333}=\frac{1}{3} \bar{P}_{33}=\frac{\gamma}{3} \\
& \bar{P}_{1111}+\bar{P}_{1212}+\bar{P}_{1313}=\frac{2}{5} \bar{P}_{11}=\frac{1-\gamma}{5} \\
& \left.2 \bar{P}_{1313}+\bar{P}_{3333}\right)=\frac{2}{5} \bar{P}_{33}=\frac{2 \gamma}{5}
\end{aligned}
$$

One has only to compute one component of $\overline{\mathbb{P}}$ for having all other ones. For instance we compute $\bar{P}_{3333}$ and we choose to define it as $2 \mu$ :

$$
\bar{P}_{3333}=2 \mu=\frac{1}{S_{1}} \int_{\varphi=0}^{\varphi=\pi} \int_{\theta=0}^{\theta=2 \pi} 2\left(1-w^{2}\right)^{2} b_{1} L_{\lambda_{1}} \sin (\varphi) d \varphi
$$

Expression of $\mu$ is given by (48). After some algebraic calculations, it is easy to compute all the components of tensor $\overline{\mathbb{P}}$ from (B.4) and to put the results in the form given by (47) where the tensor of the Walpole basis are recall below:

$$
\left\{\begin{array}{l}
\mathbb{E}_{1}=\frac{1}{2} \boldsymbol{I}_{t} \otimes \boldsymbol{I}_{t}, \quad \mathbb{E}_{2}=\boldsymbol{I}_{n} \otimes \boldsymbol{I}_{n} \\
\mathbb{E}_{3}=\boldsymbol{I}_{t} \underline{\bar{\otimes}} \boldsymbol{I}_{t}-\mathbb{E}_{1}, \quad \mathbb{E}_{4}=\boldsymbol{I}_{t} \bar{\otimes} \boldsymbol{I}_{n}+\boldsymbol{I}_{n} \underline{\bar{\otimes}} \boldsymbol{I}_{t} \\
\mathbb{E}_{5}=\boldsymbol{I}_{n} \otimes \boldsymbol{I}_{t}, \quad \mathbb{E}_{6}(\underline{\xi})=\boldsymbol{I}_{t} \otimes \boldsymbol{I}_{n}
\end{array}\right.
$$

Finally, the components of tensor $\mathbb{S}$, given by (50), in the Walpole basis, are:

$$
\mathbb{S}=\sum_{n} s_{n} \mathbb{S}_{n}
$$

with: 


$$
\left\{\begin{aligned}
s_{1}= & 3 \gamma\left[1-3 \alpha_{1}+3 f \alpha_{2}\right]\left[1-\alpha_{1}+f \alpha_{2}\right] \\
& +\mu\left(1-3 \alpha_{1}+3 f \alpha_{2}\right)^{2} \\
& +6\left[1-\alpha_{1}+f \alpha_{2}\right]\left[\alpha_{1}-f \alpha_{2}\right] \\
s_{2}= & \frac{3 \gamma}{2}\left[1-3 \alpha_{1}-3 f\left(1-\alpha_{2}\right)\right]\left[1-\alpha_{1}-f\left(1-\alpha_{2}\right)\right] \\
& +\frac{\mu}{2}\left(1-3 \alpha_{1}-3 f\left(1-\alpha_{2}\right)\right)^{2} \\
& +3\left[\alpha_{1}+\left(1-\alpha_{2}\right) f\right]\left[1-\alpha_{1}-f\left(1-\alpha_{2}\right)\right] \\
s_{3}= & f^{2}\left(\gamma+\frac{\mu}{2}\right) \\
s_{4}= & f^{2}\left(\frac{5}{2}(1-\gamma)-2 \mu\right) \\
s_{5}= & \frac{3 \gamma}{2}\left[1-3 \alpha_{1}-3 f\left(1-\alpha_{2}\right)\right]\left[1-\alpha_{1}+f \alpha_{2}\right]+\frac{3 f \gamma}{2} \\
& +\frac{\mu}{2}\left[1-3 \alpha_{1}+3 f \alpha_{2}\right]\left[1-3 \alpha_{1}-3 f\left(1-\alpha_{2}\right)\right] \\
& +3\left[\alpha_{1}+f\left(1-\alpha_{2}\right)\right]\left[1-\alpha_{1}+f \alpha_{2}\right]-\frac{3 f}{2} \\
s_{6}= & s
\end{aligned}\right.
$$

\section{Expression of functions $\mathcal{G}_{n}\left(\xi_{1}, \xi_{2}, \xi_{3}\right)$}

Starting from:

$$
\left\{\begin{aligned}
\frac{\partial \Pi}{\partial D_{n}}(\boldsymbol{D})= & \sigma_{0} f\left[\operatorname{arcsinh}\left(\frac{u Y}{X}\right)\right]_{u=u_{1}}^{u=u_{2}} \frac{\partial Y}{\partial D_{n}} \\
& -\sigma_{0} f\left[\frac{\sqrt{X^{2}+u^{2} Y^{2}}}{X}\right]_{u=u_{1}}^{u=u_{2}} \frac{\partial X}{\partial D_{n}}, \\
& +\frac{\tau_{r} S_{1}}{V_{1}} \frac{\partial W}{\partial D_{n}}+\frac{\tau_{0} S_{1}}{V_{1}} \frac{\partial Z}{\partial D_{n}}
\end{aligned}\right.
$$

with $n=m, q, s, t$ and: 


$$
\begin{aligned}
& W=g_{1} D_{m}+g_{2} D_{q} \\
& Z=\sqrt{h_{1} D_{m}^{2}+h_{2} D_{q}^{2}+2 h_{3} D_{m} D_{q}+h_{4} D_{s}^{2}+h_{5} D_{t}^{2}}
\end{aligned}
$$

and $X, Y$ are given by (31). By denoting $\xi_{n}$ the triplet $\left(\xi_{1}, \xi_{2}, \xi_{3}\right)$, it follows that:

$$
\left\{\begin{aligned}
\mathcal{G}_{1}\left(\xi_{n}\right)= & \frac{\sigma_{0} \mathcal{U}\left(\xi_{n}\right)}{f \kappa}\left[1+\frac{\eta}{3}\left(1-3 \alpha_{2}\right)\right] \\
& -\frac{1}{3} \sigma_{0}(1-\zeta) \mathcal{V}\left(\xi_{n}\right)\left[\xi_{1}+\left(1-3 \alpha_{2}\right)\right]\left(1-3 \alpha_{2}\right) \\
& +\frac{\tau_{r} S_{1}}{3 V_{1}} g_{1}+\frac{\tau_{0} S_{1}}{3 V_{1}} \frac{h_{1}+h_{3} \xi_{1}}{\mathcal{Z}\left(\xi_{n}\right)} \\
\mathcal{G}_{2}\left(\xi_{n}\right)= & \frac{\sigma_{0} \eta \mathcal{U}\left(\xi_{n}\right)}{f \kappa}-\sigma_{0}(1-\zeta) \mathcal{V}\left(\xi_{n}\right)\left[\xi_{1}+\left(1-3 \alpha_{2}\right)\right] \\
& +\frac{\tau_{r} S_{1}}{V_{1}} g_{2}+\frac{\tau_{0} S_{1}}{V_{1}} \frac{h_{2} \xi_{1}+h_{3}}{\mathcal{Z}\left(\xi_{n}\right)} \\
\mathcal{G}_{3}\left(\xi_{n}\right)=- & \sigma_{0} \mathcal{V}\left(\xi_{n}\right) \xi_{2}+\frac{\tau_{0} S_{1}}{V_{1}} \frac{h_{4}}{\mathcal{Z}\left(\xi_{n}\right)} \xi_{2} \\
\mathcal{G}_{4}\left(\xi_{n}\right)= & -\sigma_{0} \mathcal{V}\left(\xi_{n}\right) \xi_{3}+\frac{\tau_{0} S_{1}}{V_{1}} \frac{h_{5}}{\mathcal{Z}\left(\xi_{n}\right)} \xi_{3}
\end{aligned}\right.
$$

with:

$$
\left\{\begin{array}{l}
\mathcal{U}\left(\xi_{n}\right)=f\left[\operatorname{arcsinh}\left(\frac{u \mathcal{Y}\left(\xi_{n}\right)}{\mathcal{X}\left(\xi_{n}\right)}\right)\right]_{u_{1}}^{u_{2}} \\
\mathcal{V}\left(\xi_{n}\right)=f\left[\frac{\sqrt{\mathcal{X}^{2}\left(\xi_{n}\right)+u^{2} \mathcal{Y}^{2}\left(\xi_{n}\right)}}{u \mathcal{X}^{2}\left(\xi_{n}\right)}\right]_{u_{1}}^{u_{2}}
\end{array}\right.
$$

and:

$$
\left\{\begin{array}{l}
\mathcal{X}\left(\xi_{n}\right)=\left[(1-\zeta)\left(\xi_{1}+\left(1-3 \alpha_{2}\right)\right)^{2}+\xi_{2}^{2}+\xi_{3}^{2}\right]^{1 / 2} \\
\mathcal{Y}\left(\xi_{n}\right)=\frac{1}{f \kappa}\left[3+\eta\left(1-3 \alpha_{2}\right)\right]+\frac{\eta}{f \kappa} \xi_{1} \\
\mathcal{Z}\left(\xi_{n}\right)=\sqrt{h_{1}+h_{2} \xi_{1}^{2}+2 h_{3} \xi_{1}+h_{4} \xi_{2}^{2}+h_{5} \xi_{3}^{2}}
\end{array}\right.
$$

Consider the particular case of a spherical nanovoids for which the different coefficients, which enters into the definition of functions $\mathcal{G}_{1}\left(\xi_{n}\right), \mathcal{G}_{2}\left(\xi_{n}\right), \mathcal{G}_{3}\left(\xi_{n}\right), \mathcal{G}_{4}\left(\xi_{n}\right)$, 
read:

$$
\begin{aligned}
& \eta=\zeta=0, \quad \kappa=\frac{3}{2}, \quad \alpha_{2}=\frac{1}{3}, \quad g_{1}=2, \quad g_{2}=0, \\
& h_{1}=4, \quad h_{2}=h_{3}=h_{4}=\frac{3 f^{2}}{5}, \quad S_{1}=4 \pi a_{1}^{2}, \quad V_{1}=\frac{4}{3} \pi a_{1}^{3}
\end{aligned}
$$

Consequently:

$$
\left\{\begin{array}{l}
\mathcal{G}_{1}\left(\xi_{n}\right)=\frac{2 \sigma_{0} \mathcal{U}(\xi)}{3 f}+\frac{2 \tau_{r} S_{1}}{3 V_{1}}+\frac{\tau_{0}}{a_{1}} \frac{4}{\sqrt{4+\frac{3 f^{2}}{5}} \xi^{2}} \\
\mathcal{G}_{2}\left(\xi_{n}\right)=-\sigma_{0} \mathcal{V}(\xi) \xi_{1}+\frac{\tau_{0}}{a_{1}} \frac{9 f^{2}}{5 \sqrt{4+\frac{3 f^{2}}{5} \xi^{2}}} \xi_{1} \\
\mathcal{G}_{3}\left(\xi_{n}\right)=-\sigma_{0} \mathcal{V}(\xi) \xi_{2}+\frac{\tau_{0}}{a_{1}} \frac{9 f^{2}}{5 \sqrt{4+\frac{3 f^{2}}{5}} \xi^{2}} \xi_{2} \\
\mathcal{G}_{4}\left(\xi_{n}\right)=-\sigma_{0} \mathcal{V}(\xi) \xi_{3}+\frac{\tau_{0}}{a_{1}} \frac{9 f^{2}}{5 \sqrt{4+\frac{3 f^{2}}{5}} \xi^{2}} \xi_{3}
\end{array}\right.
$$

in which:

$$
\left\{\begin{array}{l}
\mathcal{U}(\xi)=f\left[\operatorname{arcsinh}\left(\frac{2 u}{f \xi}\right)\right]_{f}^{1} \\
\mathcal{V}(\xi)=f\left[\frac{\sqrt{\xi^{2}+\frac{4 u^{2}}{f^{2}}}}{u \xi^{2}}\right]_{f}^{1}
\end{array}\right.
$$

with $\xi=\sqrt{\xi_{1}^{2}+\xi_{2}^{2}+\xi_{3}^{2}}$. The mean stress $\Sigma_{m}$ is given $\Sigma_{m}=\mathcal{G}_{1}\left(\xi_{n}\right)\left(\mathcal{G}_{1}\left(\xi_{n}\right)\right.$ being given by the first relation in (C.7)). The equivalent stress $\Sigma_{e q}$ is obtained as a combination of the last three equations in (C.7):

$$
\begin{aligned}
\Sigma_{e q} & =\sqrt{\left[\mathcal{G}_{2}\left(\xi_{n}\right)\right]^{2}+\left[\mathcal{G}_{3}\left(\xi_{n}\right)\right]^{2}+\left[\mathcal{G}_{4}\left(\xi_{n}\right)\right]^{2}} \\
& =-\sigma_{0} \mathcal{V}(\xi) \xi+\frac{\tau_{0}}{a_{1}} \frac{9 f^{2}}{5 \sqrt{4+\frac{3 f^{2}}{5}} \xi^{2}} \xi
\end{aligned}
$$




\section{Derivation of the macroscopic criterion for $\tau_{0}=0$}

We put $\tau_{0}=0$ in the expressions of functions $\mathcal{G}_{1}\left(\xi_{n}\right), \mathcal{G}_{2}\left(\xi_{n}\right), \mathcal{G}_{3}\left(\xi_{n}\right), \mathcal{G}_{4}\left(\xi_{n}\right)$ given by (C.3). It leads to:

$$
\left\{\begin{aligned}
\mathcal{G}_{1}\left(\xi_{n}\right)= & \frac{\sigma_{0} \mathcal{U}\left(\xi_{n}\right)}{f \kappa}\left[1+\frac{\eta}{3}\left(1-3 \alpha_{2}\right)\right] \\
& -\frac{1}{3} \sigma_{0}(1-\zeta) \mathcal{V}\left(\xi_{n}\right)\left[\xi_{1}+\left(1-3 \alpha_{2}\right)\right]\left(1-3 \alpha_{2}\right)+\frac{\tau_{r} S_{1}}{3 V_{1}} g_{1} \\
\mathcal{G}_{2}\left(\xi_{n}\right)= & \frac{\sigma_{0} \eta \mathcal{U}\left(\xi_{n}\right)}{f \kappa}-\sigma_{0}(1-\zeta) \mathcal{V}\left(\xi_{n}\right)\left[\xi_{1}+\left(1-3 \alpha_{2}\right)\right]+\frac{\tau_{r} S_{1}}{V_{1}} g_{2} \\
\mathcal{G}_{3}\left(\xi_{n}\right)= & -\sigma_{0} \mathcal{V}\left(\xi_{n}\right) \xi_{2} \\
\mathcal{G}_{4}\left(\xi_{n}\right)= & -\sigma_{0} \mathcal{V}\left(\xi_{n}\right) \xi_{3}
\end{aligned}\right.
$$

Let us introduce:

$$
\begin{aligned}
& \mathcal{G}_{1}^{*}\left(\xi_{n}\right)=\mathcal{G}_{1}\left(\xi_{n}\right)-\frac{\tau_{r} S_{1}}{3 V_{1}} g_{1} \\
& \mathcal{G}_{2}^{*}\left(\xi_{n}\right)=\mathcal{G}_{2}\left(\xi_{n}\right)-\frac{\tau_{r} S_{1}}{V_{1}} g_{2}
\end{aligned}
$$

From the two first relations in (D.1), it can be easily shown that:

$$
\mathcal{U}\left(\xi_{n}\right)=\frac{f \kappa}{\sigma_{0}}\left[\mathcal{G}_{1}^{*}\left(\xi_{n}\right)-\frac{1}{3}\left(1-3 \alpha_{2}\right) \mathcal{G}_{2}^{*}\left(\xi_{n}\right)\right]
$$

Note also that, from equations (55) and (56), one has:

$$
\mathcal{G}_{1}^{*}\left(\xi_{n}\right)=\Sigma_{m}-C_{m}, \quad \mathcal{G}_{2}^{*}\left(\xi_{n}\right)=\Sigma_{q}-C_{q}
$$

It follows that (D.3) can be rewritten as:

$$
\mathcal{U}\left(\xi_{n}\right)=\frac{f \kappa T}{\sigma_{0}}
$$


with $T=\Sigma_{m}-C_{m}-\frac{1}{3}\left(1-3 \alpha_{2}\right)\left(\Sigma_{q}-C_{q}\right)$. Using again relations (55) and (56), the last three equations in (D.1) can be read:

$$
\left\{\begin{array}{l}
\Sigma_{q}-C_{q}-\eta T=-\sigma_{0}(1-\zeta) \mathcal{V}\left(\xi_{n}\right)\left[\xi_{1}+\left(1-3 \alpha_{2}\right)\right] \\
\Sigma_{s}=-\sigma_{0} \mathcal{V}\left(\xi_{n}\right) \xi_{2} \\
\Sigma_{t}=-\sigma_{0} \mathcal{V}\left(\xi_{n}\right) \xi_{3}
\end{array}\right.
$$

which, by combination, leads to (see equations (C.4) and (C.5) for the definitions of $\mathcal{V}\left(\xi_{n}\right)$ and $\left.\mathcal{X}\left(\xi_{n}\right)\right)$ :

$$
\mathcal{V}\left(\xi_{n}\right) \mathcal{X}\left(\xi_{n}\right)=\frac{1}{\sigma_{0}}\left[\frac{\left(\Sigma_{q}-C_{q}-\eta T\right)^{2}}{1-\zeta}+\Sigma_{s}^{2}+\Sigma_{t}^{2}\right]^{1 / 2}
$$

The last step of the demonstration consist to use the following identity:

$$
\mathcal{V}^{2}\left(\xi_{n}\right) \mathcal{X}^{2}\left(\xi_{n}\right)+\frac{2 f^{2}}{u_{1} u_{2}} \cosh \left(\frac{\mathcal{U}\left(\xi_{n}\right)}{f}\right)-\frac{f^{2}}{u_{1}^{2}}-\frac{f^{2}}{u_{2}^{2}}=0
$$

which is obtained by eliminating $\mathcal{Y}\left(\xi_{n}\right)$ in (C.4). Replacing $u_{1}$ and $u_{2}$ by their expressions given by (A.4) and considering relations (D.5) and (D.7), it easy to recover (58). 